\title{
To cope with drought: two forage grass species - Festuca arundinacea and F. glaucescens can activate similar survival strategies although they differ with molecular response
}

\section{Katarzyna Lechowicz}

Institute of Plant Genetics, Polish Academy of Sciences

Izabela Pawlowicz ( $\square$ ipaw@igr.poznan.pl)

Polska Akademia Nauk Instytut Oceanologii https://orcid.org/0000-0002-0790-6599

Dawid Perlikowski

Institute of Plant Genetics, Polish Academy of Sciences

Magdalena Arasimowicz-Jelonek

Uniwersytet im Adama Mickiewicza w Poznaniu Wydzial Anglistyki

Joanna Majka

Institute of Plant Genetics, Polish Academy of Sciences

Adam Augustyniak

Institute of Plant Genetics, Polish Academy of Sciences

Arkadiusz Kosmala

Institute of Botany Chinese Academy of Sciences

Research article

Keywords: Antioxidant enzymes, Drought strategies, Festuca arundinacea, F. glaucescens, ROS, Photosynthesis

Posted Date: December 5th, 2019

DOl: https://doi.org/10.21203/rs.2.18305/v1

License: (9) This work is licensed under a Creative Commons Attribution 4.0 International License. Read Full License 


\section{Abstract}

Background: Photosynthesis is among the primary processes affected by drought and its disturbances result with the reduction of growth, reactive oxygen species (ROS) overproduction, and alterations in antioxidant system activity. Our study was performed on two closely related forage grasses: Festuca arundinacea and F. glaucescens. Two genotypes within each species significantly differing with the potential of drought resistance: high drought resistant (HDR) and low drought resistant (LDR), were used. The research involved: (i) the analysis of gene expression at transcript and protein levels for the selected enzymes of Calvin cycle (fructose-1,6-bisphosphate aldolase (pFBA), phosphoglycerate kinase (PGK) and glyceraldehyde-3-phosphate dehydrogenase (GAPDH)) and (ii) the activity of pFBA, as a protein marker of the Calvin cycle, (iii) the analysis of gene expression at protein level for the selected antioxidant enzymes (glutathione reductase (GR), glutathione peroxidase (GPX), L-ascorbate peroxidase (APX), catalase (CAT), and superoxide dismutase (SOD)), (iv) the measurements of physiological parameters describing a plant's physiological status under control, drought and re-watering conditions (relative water content (RWC), electrolyte leakage (EL), lipid peroxidation, chlorophyll fluorescence, gas exchange, and ROS level).

Results: Our analysis clearly showed that physiological reactions to water deficit were similar in both HDR and both LDR genotypes of Festuca arundinacea and F. glaucescens but the species revealed significant differences in the potential to tolerate tissue dehydration, what was correlated with distinct expression of the Calvin cycle enzymes under drought stress.

Conclusions: The maintenance of stable efficiency of dark phase of photosynthesis seems to be crucial for drought tolerance and recovery in F. glaucescnes, whereas acquisition of drought tolerance in $F$. arundinacea and F. glaucescens does not involve marked changes at the protein level in the enzymatic antioxidant system.

\section{Background}

A lack of water is one of the main environmental factors that affects plant growth and development, and significantly reduces the yield of many crop species with economic importance. Considering rapid climate changes with global warming being the most noticeable, we can expect that periods of drought will occur more often than previously, even in temperate regions (1). Thus, the recognition of cellular mechanisms conferring plant drought resistance seems to be crucial.

Grasslands cover around $70 \%$ of the world's agricultural area and are mainly used for fodder purposes (2). Forage grasses belonging to Lolium and Festuca genera are one of the most important in the temperate regions. Our previous studies revealed that Festuca species could be successfully used as models to precisely dissect mechanisms of resistance to a wide range of abiotic stresses in a group of forage grasses (e.g. 3, 4, 5, 6, 7, 8, 9). 
Allohexaploid Festuca arundinacea Schreb. $(2 n=6 x=42)$ and tetraploid $F$. arundinacea var. glaucescens Boiss. (hereafter recognized as F. glaucescens) $(2 n=4 x=28)$ are closely related cool-season forage grasses (10) with a relatively high potential to withstand periods of water shortage. In $F$. arundinacea, drought resistance is mostly manifested by a drought avoidance strategy which relies on the development of deep root system, leaf rolling and a rapid stomatal closure $(5,11)$. However, our earlier experiments revealed that $F$. arundinacea could also develop a drought tolerance strategy mainly associated with the adjustment of leaf metabolism to water deficit in plant tissues $(5,6)$. On the other hand, F. glaucescens was described earlier as a species characterized mainly by a metabolism slowdown and a reduction of growth associated with a quiescence under drought conditions, followed by a further recovery after stress cessation, which enables it to survive and to resume the growth following irrigation $(12,13)$. The knowledge about molecular basis of drought resistance is strongly limited in this species.

Photosynthesis is among the primary processes to be affected by drought. Its efficiency can be restricted by both stomatal and non-stomatal mechanisms. Stomata closing declines $\mathrm{CO}_{2}$ availability, whereas among the non-stomatal mechanism, a reduction of Calvin cycle efficiency was indicated to be crucial (8, $14,15,16)$.

Drought, especially the initial phase of stress duration, can also result in the oxidative stress manifested by the reactive oxygen species (ROS) overproduction $(17,18)$, with hydrogen peroxide $\left(\mathrm{H}_{2} \mathrm{O}_{2}\right)$, hydroxyl radical $(\mathrm{OH})$ and superoxide anions $\left(\mathrm{O}_{2}{ }^{\circ}\right)$ being the most severe (19). Although these molecules serve as signals in the numerous processes associated with plant growth and development, ROS are also recognized as key players in stress signaling $(20,21)$. However, a stress-induced overproduction of ROS can lead to the damage of photosynthetic apparatus and the disturbance of different metabolic pathways $(21,22,23,24)$. Furthermore, the enhanced production of ROS during drought supports lipid peroxidation, and finally contributes to the damage of cellular membranes (25). The ROS-scavenging enzymatic system was proved to be one of the most crucial components of drought resistance in many plant species $(26,27)$. Moreover, the enzymes contribute to the maintenance of cellular redox balance, particularly during stress response $(28,29,30)$. The systems includes glutathione reductase (GR), glutathione peroxidase (GPX), L-ascorbate peroxidase (APX), catalase (CAT), and superoxide dismutase (SOD). Superoxide dismutases are classified according to their metal cofactor, and among them, Fedependent superoxide dismutase (Fe-SOD), $\mathrm{Cu} / \mathrm{Zn}$ superoxide dismutase ( $\mathrm{Cu} / \mathrm{Zn}-\mathrm{SOD}$ ), and manganese superoxide dismutase (Mn-SOD), are distinguished. Under water deficit, the up-regulated expression of genes encoding the antioxidant enzymes both at the transcriptional and post-transcriptional level as well as the enhancement of enzyme activities, were observed $(30,31,32)$.

Herein, we hypothesize that under water deficit in specific environmental conditions, especially those preventing the development of deep root system $F$. arundinacea and $F$. glaucescens could cope with this stress activating a set of similar survival strategies, involving mostly drought tolerance and recovery after stress cessation. We also assume that these strategies will be manifested at the levels of both photosynthetic performance and cellular antioxidant system. Thus, the comprehensive research presented in this paper involved $(i)$ the analysis of gene expression at transcript and protein levels for the 
selected enzymes of Calvin cycle (fructose-1,6-bisphosphate aldolase (pFBA), phosphoglycerate kinase (PGK) and glyceraldehyde-3-phosphate dehydrogenase (GAPDH)) and (ii) the activity of pFBA, as a protein marker of the Calvin cycle, (iii) the analysis of gene expression at protein level for the selected antioxidant enzymes (GR, GPX, APX, CAT, Fe-SOD, Cu/Zn-SOD, and Mn-SOD), (iv) the measurements of a set of physiological parameters precisely describing a plant's physiological status under control, drought and re-watering conditions. These parameters included a relative water content (RWC), electrolyte leakage (EL), lipid peroxidation, chlorophyll fluorescence, gas exchange, and level of ROS generations.

\section{Results}

\section{Genomic structure of $F$. arundinacea and $F$. glaucescens}

Allotetraploid F. arundinacea had 42 chromosomes, while tetraploid F. glaucescens had 28 chromosomes in total, respectively. Our results indicate that, the hybridization of $5 S$ rDNA revealed six signals in chromosomes of the HDR and the LDR genotypes of $F$. arundinacea and four signals in both genotypes of $F$. glaucescens (Fig. 1, red signals). In all the analysed plants, 5S rDNA loci were located in the interstitial part of chromosomes. The results of fluorescent in situ hybridization (FISH) using 35S rDNA sequence as a probe showed the same number of signals in both genotypes of $F$. arundinacea and $F$. glaucescens (Fig. 1, green signals). However, these four signals were distributed in a different way in these two species. In $F$. arundinacea karyotypes, one pair of chromosomes with the terminal position of 35S rDNA and one pair with the interstitial position of this sequence (Fig. 1A and B), were observed. For both $F$. glaucescens genotypes, only the terminal distribution of 35S rDNA was noticed (Fig. 1C and D).

\section{Phenotypic observations under drought and recovery}

Under severe drought stage a leaf rolling phenomenon was noticeable in both genotypes of $F$. arundinacea and F. glaucescens. After rehydration, the leaves returned to their usual shape in all the analyzed genotypes, nevertheless numerous of dried leaves could be observed (Fig. 2). Both $F$. glaucescens genotypes had stronger growth inhibition during water deficit, compared with the $F$. arundinacea genotypes.

\section{Physiological parameters}

The significant drop of RWC was observed on the $11^{\text {th }}$ day (D3) of stress treatment comparing to the control conditions (value 95-98\%) in all the analyzed genotypes. However, in the LDR genotypes of both Festuca species (Fa-LDR and Fg-LDR) RWC reduction was notably larger (value around $40 \%$ ) than in both HDR genotypes (Fa-HDR and Fg-HDR) (value around 70\%). After further re-watering RWC increased to the level similar to the control conditions in all genotypes. Both, in the control conditions, initial phase of 
drought (D1, D2) and after stress cessation there were no significant differences in RWC dynamic between the F. arundinacea and F. glaucescens genotypes (Fig. 3A).

The values of EL did not change during the whole drought period in both HDR genotypes of both Festuca species but it significantly increased on the $11^{\text {th }}$ day (D3) of water deficit in both LDR genotypes. Much higher EL growth was noted for the Fg-LDT genotype. However, after re-watering, the EL values decreased to the control level in both LDR genotypes (Fig. 3B).

In the severe drought (D3) both analyzed genotypes of $F$. arundinacea and $F$. glaucescens reduced significantly, to a similar level, their $\mathrm{CO}_{2}$ assimilation rate $(A)$, stomatal conductance $\left(g_{s}\right)$ and transpiration (E) values. Interestingly, in the control conditions higher $A$ and $g_{s}$ were reported for the Fa$\mathrm{HDR}$ genotype and the Fg-LDR genotype. After re-watering, $\mathrm{CO}_{2}$ assimilation rate increased slightly but significantly only in the Fg-LDR genotype, while transpiration was higher in comparison to the drought conditions only in the Fa-LDR genotype (Fig. 4A, B and C). The intracellular concentration of $\mathrm{CO}_{2}(\mathrm{Ci})$ increased significantly in all the genotypes during the stress treatment. After further re-watering, a reduction of this parameter was observed in all the genotypes, except the Fg-HDR genotype (Fig. 4D).

In the Fa-HDR genotype, the majority of parameters of chlorophyll fluorescence remained stable or decreased during prolonged drought. Values of the following parameters: ABS/RC, ABS/CS, TRO/RC and TRO/CS demonstrated significant differences between the Fa-HDR and Fa-LDR genotypes, but only at D3 time-point of drought stress. The ABS/RC, ABS/CS and TRO/RC increased significantly in the Fa-LDR genotype in the advanced drought (Fig. S1A, B, C). ET0/CS parameter declined notably, in both $F$. arundinacea genotypes and slightly increased after re-watering (Fig. S1E). No significant changes in all the analyzed fluorescence parameters between the Fa-LDR and Fa-HDR genotypes after stress cessation, were noticed (Fig. S1, Fig. S2). Significant differences between the Fg-HDR and Fg-LDR genotypes of $F$. glaucescens on the $11^{\text {th }}$ day (D3) of drought were observed in numerous parameters (ET0/Cs, RC/CSm, DIO/RC, DIO/CS) (Fig. S1E, Fig. S2A, C, D). These changes were resulted from a decrease or increase in the values of these parameters in the Fg-LDR genotype at D3 time-point. After recovery, in the Fg-LDR genotype, all the changed parameters returned to values observed in the control conditions or in the initial phase of stress. In several measured parameters of chlorophyll fluorescence (ABC/RC, ETO/CS, RC/CSm, $\mathrm{RC} / \mathrm{CSO}, \mathrm{DIO} / \mathrm{RC}$ ) significant differences were visible between the Fg-HDR and Fg-LDR genotypes after drought cessation (Fig. S1A, E, Fig. S2A, B, C). These differences were mainly due to a reduction of the values observed in the Fg-HDR genotype and an increase in the Fg-LDR genotype. Only in case of $A B S / R C, T R 0 / R C$ and DIO/RC, an increase was observed in the Fg-HDR genotype at RH time-point (Fig. S1A, C, Fig. S2C).

\section{Expression of genes encoding the Calvin cycle enzymes at transcript and protein levels}


The transcription profiles patterns of PGK and pFBA were similar in both $F$. arundinacea genotypes (FaHDR and Fa-LDR). After initial increase on the $3^{\text {rd }}$ day of drought (D1), decrease to the control level was observed. No changes in transcript accumulation, comparing to the control in analyzed genotypes, were noticed after re-watering. However, clear differences between the genotypes (Fa-HDR and Fa-LDR) in PGK and pFBA transcript level were visible after re-watering $(\mathrm{RH})$ and on the $3^{\text {rd }}$ day of drought (D1), respectively. The control level of PGK transcript was equal in both F. glaucescens genotypes, whereas pFBA transcript accumulation was about twofold higher in the Fg-HDR than in Fg-LDR genotype. Under water deficit, transcript level of both PGK and pFBA significantly decreased in the Fg-HDR and Fg-LDR genotypes, achieving the lowest values on the $11^{\text {th }}$ day of stress (D3). After rehydration PGK level was significantly higher comparing to the control in both genotypes, whereas pFBA level only in the Fg-LDR genotype. The clear differences under stress conditions between the F. glaucescens genotypes in PGK and pFBA transcript abundance were observed at D1 and D2, respectively (Fig. 5A, B).

GAPDH transcript level increased on the $11^{\text {th }}$ day (D3) of water deficit, comparing to the control in both $F$. arundinacea genotypes and it was significantly higher in the Fa-HDR genotype. After stress cessation it returned to the initial level. In the HDR genotype of $F$. glaucescens GAPDH level remained relatively stable during whole drought period, however it increased about twofold after re-watering. In the Fg-LDR genotype a slight drop of transcript level was observed at D2 time-point. During the whole period of experiment the level of GAPDH was higher in the Fg-LDR genotype than in the Fg-HDR genotype (Fig. 5C).

A significant growth of PGK protein level at the beginning of stress duration (between D1-D2) was observed in the LDR genotype of $F$. arundinacea. In the advanced drought (D3) it decreased to the lower level than observed in the control conditions, while in the recovery phase it returned to the initial level. In the Fa-HDR genotype PGK protein level decreased in the late stage of stress (D2 and D3). The increase of accumulation level of PGK protein in both genotypes of $F$. glauacescens was observed during the first six days of drought duration. Then its level decreased on the $11^{\text {th }}$ day (D3) of drought and increased back after stress cessation in both genotypes. However, only in Fg-HDR plants the accumulation exceeded the control (Fig. 6A).

No relevant changes in the pFBA accumulation level in the Fa-LDR genotype during the whole experiment, were observed. In the Fa-HDR genotype, a slight increase was noticed on the $3^{\text {rd }}$ and $11^{\text {th }}$ day of water deficit. However, that accumulation level dropped again after rehydration to the values observed in the control conditions. The accumulation level of pFBA protein in the Fg-HDR genotype increased from the initial time-points of drought duration (D1-D2) to the rehydration time-point (RH). Interestingly, its level was constant during the whole experiment in the Fg-LDR genotype (Fig. 6B).

The amount of GAPDH protein was more or less constant during drought treatment in two $F$. arundinacea genotypes, and it dropped after rehydration. The similar tendency was observed for $F$. glaucescens genotypes, however, after re-watering, the amount of GAPDH decreased only in the Fg-LDR genotype (Fig. $6 \mathrm{C})$. 


\section{Chloroplast aldolase activity}

The activity of pFBA decreased in both $F$. arundinacea genotypes in response to drought. In the Fa-HDR genotype lowered activity was observed during the whole stress period, whereas in the Fa-LDR genotype only on the $11^{\text {th }}$ (D3) day of drought. In the recovery phase pFBA activity increased to the control level only in the Fa-LDR genotype. In F. glaucescens genotypes, a significant decline of pFBA activity was observed between the $6^{\text {th }}$ (D2) and $11^{\text {th }}$ (D3) day of drought, compared to the control. After re-watering an increase of pFBA activity comparing to the advanced water deficit (D3) was revealed for both $F$. glaucescens genotypes, but it was lower than in the control. The genotypes differed with pFBA activity during the whole stress period, that was higher in the Fg-HDR genotype (Fig. 7).

\section{Expression of genes coding antioxidant enzymes at the protein level}

The protein accumulation of Cu/Zn-SOD was rather stable during the whole experiment in both Festuca species. Its slight reduction in the initial phase of stress duration (D1) and higher accumulation, compared to control at D3, were observed in the Fa-HDR genotype and Fg-LDR genotypes, respectively. The lowering of its level was also noticeable in both $F$. arundinacea genotypes after re-hydration. In the control conditions the protein level of Mn-SOD was about twofold higher in the Fa-HDR genotype than in the Fa-LDR genotype. During the whole drought period, its level was elevated in the Fa-LDR, and then it dropped and returned to the control level after stress cessation $(\mathrm{RH})$. In the Fa-HDR genotype, the reduced protein level of Mn-SOD in D2 and after re-watering, was noticed. A significant Mn-SOD accumulation was observed between the $6^{\text {th }}$ and $11^{\text {th }}$ day (D2-D3) of stress duration in the Fg-HDR genotype. In the FgLDR genotype a slow declined of Mn-SOD protein level was remarked during the whole experiment in relation to control. The slight increase of Fe-SOD protein amount was observed only at the beginning of stress treatment (D1) in the Fa-HDR genotype. In the Fa-LDR genotype, a significant reduction in the amount of Fe-SOD during water deficit, was observed. After re-watering it returned to the control level. In the Fg-LDR genotype the Fe-SOD protein was highly accumulated during the stress treatment as well as after regeneration (Fig. 8A, B, C).

Stress-induced changes in APX protein accumulation were noticed in the Fa-LDR genotype. The protein level was reduced comparing to the control during the whole drought period and after re-watering. Lowered APX protein level was also observed at D2 in the Fg-HDR genotype and at D2 and D3 time-points in the Fg-LDR genotype (Fig. 9A). In the Fa-HDR genotype a statistically significant decrease of GPX, was observed during the treatment (Fig. 9B, C). In contrary, in the Fg-HDR genotype, the accumulation level of the GPX was higher under stress treatment and after re-watering. In the Fg-LDR genotype a decrease at more advanced time-points of drought (D2, D3), was observed (Fig. 9B). On the $3^{\text {rd }}$ day (D1) of water deficit a significant decrease of GR in the Fa-HDR genotype, was shown. This was the only time-point at which differences in GR amount between $F$. arundinacea genotypes, were revealed. On the other hand, the higher accumulation level of GR for the Fg-HDR, compared to the Fg-LDR genotype, was demonstrated during the whole experiment (Fig. 9C). No significant changes in CAT abundance during drought and after 
rehydration was shown for the Fa-LDR genotype. In case of the Fa-HDR genotype, the amount of this protein was elevated at three time-points (D1, D3 and RH). Significant differences in the accumulation level of CAT between F. glaucescens genotypes were visible at all the experimental time-points, and their levels were always higher in case of the Fg-HDR genotype (Fig. 9D).

\section{Lipid peroxidation}

Under water deficit TBARS accumulation increased in both $F$. arundinacea genotypes. The initiall growth in D1 and then decrease in D2 was noticed in the Fa-LDR genotype. However, the highest ca. threefold grow of TBARS was observed on the $11^{\text {th }}$ day of drought (D3) in both genotypes of $F$. arundinacea. Furthermore, this elevated level of TRARS was stable after re-watering. In the Fg-HDR genotype, TBARS accumulation level showed a downward trend reaching the lowest level at D3. On the other hand, it increased significantly at D1 and D3 time-points in the Fg-LDR genotype, but dropped again, even below the level observed in the control conditions, after stress cessation (Fig. 10A).

\section{Superoxide anion radical and hydrogen peroxide level}

The amount of superoxide anion radical decreased drastically at the beginning of water deficit (D1), compared to the control in both F. arundinacea genotypes. However, in the subsequent day of stress its level started to rise. In the Fa-HDR genotype it reached the value observed in the control on the $11^{\text {th }}$ day of drought, whereas in the Fa-LDR genotype on the day $6^{\text {th }}$ and $11^{\text {th }}$ it remained lower than in the control. The content of $\mathrm{O}_{2}{ }^{\cdot-}$ did not change after re-watering in the Fa-HDR but increased more in the Fa-LDR plant. Contrary, in the F. glaucescens genotypes, a slight reduction of $\mathrm{O}_{2}{ }^{\cdot-}$ amount at the $\mathrm{D} 1$ and $\mathrm{D} 2$ timepoints of water deficit was observed only for the Fg-LDR plants. In the advanced drought (D3) its content was significantly higher in both Fg-HDR and Fg-LDR genotypes, compared to control condition, however, without significant differences between the genotypes. Both F. glaucescens genotypes revealed diminished accumulation level of the molecule after stress cessation (Fig. 10B).

A significant reduction of hydrogen peroxide accumulation level between the control and the $6^{\text {th }}$ day (D2) of drought was reported for both $F$. arundinacea genotypes. At the D3 time-point this level was stable in the Fa-HDR genotype, whereas in the Fa-LDR its value significantly increased. A decrease of hydrogen peroxide accumulation level was observed for both $F$. arundinacea after stress cessation. The accumulation of $\mathrm{H}_{2} \mathrm{O}_{2}$ increased in the Fg-LDR genotype on the $11^{\text {th }}$ day (D3) of drought. However, at the beginning of stress treatment (D1, D2) its level slightly decreased in both genotypes of $F$. glaucescens. Furthermore, a significant reduction in $\mathrm{H}_{2} \mathrm{O}_{2}$ generation was noticed for the two genotypes but was more deeper for the Fg-LDR genotype, after stress treatment (RH) (Fig. 10C).

\section{Discussion}


The plants response to water deficiency is a combination of biological mechanisms that involve morphological, physiological and molecular adaptations. They use different strategies to survive under stress conditions that trigger several metabolic pathways at the same time (14). Acquired strategy of majority of the plant species strongly depends on the environmental conditions. Certainly, the efficient photosynthesis and the activation of enzymatic antioxidant system under drought are among important goals.

Our earlier research performed on $F$. arundinacea allowed to recognize crucial components of leaf metabolisms as well as roots performance, including its architecture and metabolism, involved in drought resistance of this species $(5,33)$. On the other hand, it has been here first time when general response to drought in F. glaucescens at the physiological as well as molecular levels was more deeply analysed. The species identities were confirmed by using FISH with rDNA probes. Both species presented the karyotypes precisely characterized earlier by Thomas et al. (1997) (34).

\section{Physiological response to drought}

The reduction of RWC could be a good indicator of cellular dehydration in plants, including grass species $(5,35)$. Our results clearly indicate that both genotypes of $F$. arundinacea and $F$. glaucescens were suffered from water deficit. However, the higher reduction of leaf RWC in the stress conditions were observed in both LDR genotypes, what could be partially due to differences in root metabolism and more efficient water uptake in case of HDR plants at the early stage of the stress (36). Our recent study proved that in case of $F$. arundinacea, deep root system is not sufficient to fully avoid cellular damage, caused by drought. In the experiment performed in tubes, thus enabling undisturbed development of root system, it was demonstrated that not only architecture but also metabolic performance of roots were crucial to cope with negative effect of water deficit by using different survival strategies (33).

The chlorophyll fluorescence parameters provides the information about the efficiency of flow of energy from antenna to the electron transport chain components through the reaction centre of photosystem II (PSII) (37). Photochemical processes were more affected in the Fa-LDR and Fg-LDR genotypes, compared to the HDR genotypes. However, both LDR genotypes showed a high capacity of regeneration after stress cessation, which resulted in a return to the levels similar to the control conditions. Interestingly, in the FgHDR genotype no significant changes during drought treatment were observed in numerous parameters, while their significant decrease after re-watering, was visible.

The physiological analysis clearly indicated that cellular response to water deficit was very similar in case of $F$. arundinacea and $F$. glaucensens, at least with respect to the analyzed parameters. Furthermore, the HDR plants of both species were able to avoid strong cellular dehydration and membrane damage at the advanced drought. On the other hand, the LDR plants of both species demonstrated a high capacity to regenerate their metabolism after stress cessation. The level of $\mathrm{CO}_{2}$ assimilation under stress and control conditions was in case of both species dependent, at least partially, on stomata aperture. 
The level of membrane integrity under drought and oxidative stress was recognized through the EL and MDA level measurements. The EL was the other physiological parameter, with similar dynamics in case of both species. It was unchanged in the HDR genotypes of both species during the whole experiment, whereas in both LDR genotypes, it was relatively high in the advanced drought conditions. However, after re-watering, it dropped to the control values. Observations for $F$. arundinacea genotypes correspond with our earlier results described by Kosmala et al. (2012) (5) and show that recovery after stress cessation is the strategy developed by both species. We demonstrated here that drought caused a lipid peroxidation in both genotypes of $F$. arundinacea, at the advanced (D3) and also at the initial stage of water deficit (D1) in the LDR. Furthermore, this parameter remained at the high level also after rehydration in both genotypes. The dynamics of lipid peroxidation in response to drought was similar only in the LDR genotype of F. glaucescens. Similarly to the EL, the MDA content in the Fg-HDR genotype only slightly decreased in the advanced drought. Interestingly, several studies indicated that the MDA level increased in green tissues of plants (citrus, maize) exposed to a combination of abiotic stresses, such as drought and heat $\left(10 \mathrm{~d}, 40^{\circ} \mathrm{C}\right)$. Furthermore, in a single stress application, the MDA did not increase or it was relatively low $(38,39,40)$. On the other hand, higher than in the control, the level of MDA after re-watering in both $F$. arundinacea genotypes and in the LDR of $F$. glaucescens indicates a more efficient functioning of defense mechanisms in the first species. Moreover, the Fg-LDR genotypes of $F$. glaucescens was a highest capacity to regenerate damaged cellular membranes.

A reduction of gas exchange parameters observed at the D3 time-point (the $11^{\text {th }}$ day of water deficit), compared to the control conditions, was similar in F. arundinacea and F. glaucescens. On the other hand, the values of these parameters were maintained at lower levels also after 10 days of watering in both species. It shows that recovery period was not sufficient in both species to bring back optimal efficiency of photosynthesis. It indicates that stomatal regulation of photosynthesis dominates in both species. It was shown here, in the analyzed genotypes of both species, that stomata closure significantly limited $\mathrm{CO}_{2}$ content. However, several earlier studies, including ours, indicated that the efficiency of the Calvin cycle could be also responsible for that phenomenon, as an important component of non-stomatal limitations of photosynthesis $(8,16,41)$.

\section{Efficiency of Calvin cycle}

In the case of presented results in both $F$. arundinacea genotypes, elevated transcript level of pFBA and PGK genes was observed at the beginning of stress duration (D1). It was correlated with the protein accumulation of those enzymes but only in the Fa-HDR genotype. In F. glaucescens, drought conditions induced accumulation of PGK in both genotypes as well as pFBA in the Fg-HDR genotype. The elevated levels of PGK and pFBA in $F$. glaucescens were also observed during recovery phase in the more resistant genotype what was not noticeable in the Fg-LDR genotype. During recovery phase, the protein level of these two enzymes was the highest in the Fg-HDR genotype. We can assume that the PGK and pFBA are involved in more efficient dark phase of photosynthesis what acquires drought tolerance. 
The up-regulation of GAPDH in $F$. arundinacea in the advanced drought, was noticed while in $F$. glaucescens drought did not significantly affect the relative expression level of this gene, which was higher in the Fg-LDR genotype.

Interestingly, the Fa-HDR and the Fg-HDR genotypes, accumulated higher amounts of pFBA protein during the whole period of water deficiency. This enzyme was shown to be crucial for the efficiency of the Calvin cycle under drought conditions in our earlier experiment performed on L. multiflorum/F. arundinacea introgression forms $(8,16)$. However, the higher abundance of pFBA during drought period was followed by its higher activity only in case of the Fg-HDR genotype. No clear and significant differences with respect to pFBA activity were revealed between analyzed here $F$. arundinacea genotypes. Thus, we can conclude that non-stomatal regulations of $\mathrm{CO}_{2}$ assimilation under drought, with respect to pFBA accumulation and activity, were not crucial in case of F. arundinacea, and even in case of F. glaucescens. Net photosynthesis in relation to all the analyzed here genotypes fully followed the levels of stomatal conductance, despite the observed differences in pFBA abundance and activity between the $F$. glaucescens genotypes.

\section{ROS production and performance of enzymatic antioxidant system}

Reactive oxygen species are involved in plant development processes. The balance between their production and scavenging plays a crucial role in a proper functioning of plants. Abiotic stresses occurrence in the environment lead to elevated ROS generation that causes cellular damage (42). However, the increase of cellular antioxidant activity could enhance the protection against oxidative damage caused by stresses, including drought $(43,44,45)$. Our results show that water deficit did not generate the high level of ROS favouring oxidative stress in the analyzed species. An initial decrease in superoxide anion radical and hydrogen peroxide release may be caused by effective functioning of antioxidant system in response to early water deficit. The prolongation of stress duration contributed to a generation of significantly higher amount of superoxide anion radical in both $F$. glaucescens genotypes. After further re-watering, the abundance of ROS dropped to levels in the control conditions or it was even lower.

The protein levels of Mn-SOD, APX, GPX, GR and CAT were significantly higher in the Fg-HDR genotype than in the Fg-LDR genotype. These results indicate that the Fg-HDR genotype exhibited higher antioxidant protein accumulation against ROS-induced oxidative stress damage than did the Fg-LDR genotype.

The superoxide dismutases with different metal co-factor are the enzymes that catalyze the dismutation of superoxide anion radical into molecular oxygen $\left(\mathrm{O}_{2}\right)$ or hydrogen peroxide $\left(\mathrm{H}_{2} \mathrm{O}_{2}\right)$. In plant cells, $\mathrm{Fe}$ SOD are located in chloroplasts, Mn-SOD in mitochondria and peroxisomes, and $\mathrm{Cu} / \mathrm{Zn}-\mathrm{SOD}$ in chloroplasts, cytosol, and possibly also in extracellular space (46). Accumulation of Fe-SOD and Mn-SOD in the Fg-LDR and Fg-HDR genotype, respectively may be associated with the compensatory mechanisms 
to counteract enhanced superoxide anion radical production in the response to drought stress in $F$. glaucescens.

Noteworthily, $\mathrm{O}_{2}^{--}$amount raised 2.5-fold in succulent purslane under heat and combined stress, but not in plants exposed to drought (40). In cotton cultivars, no significant differences in $\mathrm{H}_{2} \mathrm{O}_{2}$ levels were observed for drought and combined drought/heat stress (42). It is well known that catalase, which is involved in the degradation of $\mathrm{H}_{2} \mathrm{O}_{2}$ into water and oxygen, is the major $\mathrm{H}_{2} \mathrm{O}_{2}$-scavenging enzyme in plants (47). In our analyses, we observed a decline of $\mathrm{H}_{2} \mathrm{O}_{2}$ level throughout the experiment in the Fa-HDR genotype. We infer that this result might be due to the increase of catalase accumulation during water deficit. Furthermore, a significantly lower generation of $\mathrm{H}_{2} \mathrm{O}_{2}$ in the $\mathrm{Fg}-\mathrm{HDR}$ genotype, compared to the FgLDR genotype, was correlated with the higher accumulation level of CAT.

Interestingly, the elevated level of TBARS in both genotypes of $F$. arundinacea at D1, D3 and RH timepoints were not correlated with a higher level of $\mathrm{H}_{2} \mathrm{O}_{2}$ and superoxide anion radical. These results imply that in $F$. arundinacea and $F$. glaucescens other factors, including other ROS, contributed to the observed lipid peroxidation. Moreover, a lipoxygenase-dependent lipid peroxidation can occur to form lipid hydroperoxides (48) used e.g. as substrates for drought-induced jasmonic acid synthesis. It should be noted that the maintenance of low or moderate level of ROS during stress can facilitate their function as second messengers mediating defense/tolerance reactions in plant cells, including stomatal closure or programmed cell death (49).

\section{Conclusions}

Our analyses clearly shows that physiological reactions to water deficit were similar in both HDR and LDR genotypes of Festuca species. The HDR genotypes were able to maintain water homeostasis and membrane stability during stress treatment, whereas the LDR genotypes revealed higher recovery capacity after stress cessation. This clearly shows that physiological reaction to drought was similar in both species. However, $F$. arundinacea and $F$. glaucescens revealed significant differences in the potential to tolerate tissue dehydration what was correlated with a distinct expression level of the Calvin cycle enzymes under stress. It was showed that in F. glaucescens PGK and pFBA expression was involved in both dehydration tolerance and recovery. Moreover, maintenance of stable efficiency of dark phase of photosynthesis seems to be crucial for drought tolerance and recovery in $F$. glaucescnes. On the other hand, the acquisition of drought tolerance (tissue dehydration) in F. arundinacea and F. glaucescens does not involve marked changes at the protein level in the enzymatic antioxidant system. Presented results clearly indicated that in case of potexperimental conditions both species were characterized by a similar drought response mainly consisting of drought tolerance and recovery that was clearly indicated at the physiological level. All performed analysis were presented in the scheme (Fig. 11) which summarized the similarities and differences between all tested genotypes during water deficit as well as after rehydration.

\section{Methods}




\section{Plant material, growth conditions and experimental design}

For the analysis, two genotypes within each species, $F$. arundinacea cv. Kord $(2 n=6 x=42)$ and $F$. glaucescens ( $F$. arundinacea Schreb. subsp. Fenas (Lag.) Arcang.) $(2 n=4 x=28)$, significantly differing with the potential of drought resistance: high drought resistant (HDR) and low drought resistant (LDR), were used (Tab. 1). The drought resistance of the selected genotypes was evaluated based on the measurements of chlorophyll fluorescence (OJIP) during short-term drought treatment (11-day water deficit and further 10-day recovery) performed on the pot-planted plants growing in the environmental chamber (hereafter termed potexperiment) (5). The genotypes of $F$. arundinacea derived from single seeds originated from the collection of Institute of Plant Genetics, Polish Academy of Sciences, created by prof. Zbigniew Zwierzykowski. The seeds of $F$. glaucescens (ABY-Bn 354-1980) derived from the collection of the Institute of Biological, Environmental and Rural Sciences (IBERS) (UK), originated from the Centre de Recherches de Lusignan, INRA (France) and donated in 1985 to IBERS. This collection at IBERS was hold by the Genetic Resources Unit (Mr lan D., Thomas). The analysis of genomic structure was performed for both species, $F$. arundinacea and $F$. glaucescens, to precisely confirm their identity.

The genotypes of both Festuca species were exposed for short-term drought in the potexperiment as described previously by Kosmala et al. (2012) (5). Each genotype was represented by 15 independent clones (each three growing in a separate pot with $4 \mathrm{dm}^{3}$ of sand : peat (1:3) mixture). The conditions of experiment were as follows: temperature $-22^{\circ} \mathrm{C}, 16 \mathrm{~h}$ photoperiod, $400 \mu \mathrm{mol} \mathrm{m} \mathrm{m}^{-2} \mathrm{~s}^{-1} \mathrm{PPFD}$ (photosynthetic photon flux density), air humidity 50-60\%. Plant material (leaf tissue) for the analysis was harvested before stress treatment when plants were fully hydrated (control, C), on the $3^{\text {rd }}$ (D1), $6^{\text {th }}$ (D2) and $11^{\text {th }}$ (D3) day of watering cessation, and 10 days after subsequent re-watering (RH) (Fig. 12).

\section{Fluorescent in situ hybridization (FISH) analysis}

To verify the genomic status of $F$. arundinacea and $F$. glaucescens plants, FISH experiment with two highly conserved rDNA sequences (5S and 35S rDNA) as probes was applied. The wheat clone pTa794 containing 5S rDNA was labeled by PCR with tetramethyl-rhodamine-5-dUTP (Roche, Mannheim, Germany). Whereas, 35S rDNA, generated from a $2.3 \mathrm{~kb}$ fragment of the 25S rDNA coding region of Arabidopsis thaliana, was labeled by nick-translation with digoxigenin-11-dUTP (Roche, Mannheim, Germany). The preparation of slides, the labeling of probes and FISH experiments were performed according to protocols described in Majka et al. (2017) (50). Briefly, in FISH protocol, a hybridization mixture consisted of $50 \%$ formamide, $2 \times$ SSC, $10 \%$ dextran sulfate and $100 \mathrm{ng}$ of rDNA probes. The hybridization mixture together with the good quality of chromosome slides were denatured at $80^{\circ} \mathrm{C}$ for 2 min and then incubated overnight at $37^{\circ} \mathrm{C}$. In the protocol, it was applied fluorescein isothiocyanateconjugated (FITC) anti-digoxigenin antibody to detect digoxigenin-labeled 35S rDNA probe. After counterstaining with 4, 6-diamidino-2-phenylindole (DAPI, Sigma, St. Louis, Missouri), the slides were mounted in antifade Vectashield solution (Vector Laboratories, Burlingame, CA, USA). Slides were evaluated under an Olympus BX 61 automatic epifluorescence microscope equipped with an Olympus 
XM10 CCD camera. All images were captured using Olympus Cell-F imaging software (ver. 3.1; Olympus Soft Imaging Solutions GmbH, Germany) and Micrographx Picture Publisher software (ver. 10; Corel Corporation, Canada).

\section{Physiological parameters}

A relative water content (RWC), electrolyte leakage (EL), chlorophyll 'a' fluorescence and gas exchange (net photosynthesis ( $\mathrm{CO}_{2}$ assimilation), transpiration, stomatal conductance) were measured as described previously in detail by Kosmala et al. (2012) and Perlikowski et al. $(2014)(5,8)$. For all the physiological measurements, the second fully expanded leaves from the top of the plant were used. The RWC was calculated according to the following formula: RWC\% $=(F W-D W) /(S W-D W) \times 100$, where FW was the leaf fresh weight, DW was the leaf dry weight, and SW was the leaf turgid weight. The EL was measured using conductivity meter (Hanna Instruments EC215 Conductivity Meter) and calculated as follows: L1/L2 X 100, where L1 and L2 were electrolyte leakage of the fresh leaves and the leaves frozen in liquid nitrogen, respectively. Chlorophyll 'a' fluorescence was measured by the HandyPEA fluorimeter (Hansatech Instruments Ltd., King's Lynn, England) during midday. For RWC, EL, and chlorophyll 'a' fluorescence measurements three biological and ten technical replicates of all the analyzed genotypes at each time-points of experiment (C, D1, D2, D3, RH), were applied. Gas exchange was measured through CIRAS-2 Portable Photosynthesis System (PP SYSTEMS) at three selected time-points (C, D3, and RH) in three biological replicates.

\section{Transcript levels of Calvin cycle enzymes under drought and recovery}

RT-qPCR analyses were carried out for chloroplastic fructose-1,6-bisphosphate aldolase (pFBA), phosphoglycerate kinase (PGK) and glyceraldehyde-3-phosphate dehydrogenase (GAPDH). Total RNA was extracted from $100 \mathrm{mg}$ of the leaves using the RNeasy Plant Mini Kit (Qiagen) according to the protocol. The remaining DNA was removed from the samples using the RNase-Free DNase set (Qiagen). The cDNA was synthesized with the Maxima First Strand cDNA Synthesis Kit (Thermo Scientific). The RTqPCR assays were performed using the FastStart Essential DNA Probes Master (Roche) through the BioRad CFX 96 thermal system as described by Pawłowicz et al. (2018) (7). The reaction temperature profile was as follows: initial denaturation $95^{\circ} \mathrm{C}$ for $10 \mathrm{~min}$, followed by 44 cycles of $95^{\circ} \mathrm{C}$ for $10 \mathrm{~s}$, and $60^{\circ} \mathrm{C}$ for $30 \mathrm{~s}$ and final $50^{\circ} \mathrm{C}$ for $30 \mathrm{~s}$. The relative quantification method $(\Delta \Delta \mathrm{Cq})$ was used. The reactions were normalized using actin and ubiquitin as reference genes. The expression stability of reference genes under drought conditions was evaluated using the BestKeeper software. Primers and TaqMan probes of analyzed genes were designed through the Beacon Designer software. All the measurements were carried out in three biological and two technical replicates.

\section{Protein levels of Calvin cycle enzymes and antioxidant enzymes under drought and recovery}


Protein accumulation profiles of three enzymes of Calvin cycle (pFBA, PGK, GAPDH) and seven antioxidant enzymes including: glutathione reductase (GR, AS06 181), chloroplastic glutathione peroxidase (GPX, AS04 055), chloroplastic Fe-dependent superoxide dismutase (FeSOD, AS06 125), chloroplastic $\mathrm{Cu} / \mathrm{Zn}$ superoxide dismutase (Cu/Zn-SOD, AS06 170), manganese superoxide dismutase (Mn-SOD, AS09 524), L-ascorbate peroxidase (APX, AS08 368) and catalase (CAT, AS09 501), were analyzed. Total proteins were extracted from the leaves using Hurkman and Tanaka protocol with slight modifications $(4,51,52)$. Briefly, the $200 \mathrm{mg}$ of powdered tissue was homogenized with $500 \mu \mathrm{l}$ of extraction buffer ( $0.7 \mathrm{M}$ sucrose, $0.5 \mathrm{M}$ TRIS, $30 \mathrm{mM} \mathrm{HCl}, 50 \mathrm{mM}$ EDTA, $2 \%$ DTT, and $0.1 \mathrm{M} \mathrm{KCl}$ ). An equal volume of phenol was then added, vortexed and centrifuged at $21500 \mathrm{~g}$ for $15 \mathrm{~min}$. The upper phenol phase was transferred to new tubes and $500 \mu l$ of extraction buffer was added. After vortexing and centrifuging in the same conditions, the proteins from the phenol phase were precipitated by the addition of 5 volumes of cold $0.1 \mathrm{M}$ ammonium acetate in methanol in the new tube. After at least overnight incubation at $-20^{\circ} \mathrm{C}$, the samples were centrifuged at $9000 \mathrm{~g}$ at $0^{\circ} \mathrm{C}$ for $30 \mathrm{~min}$. The precipitate was washed once with the cold ammonium acetate in methanol and twice in cold acetone, and dried in SpeedVac (Heraeus Instruments). Dried precipitate was dissolved in $150 \mu$ of resolving buffer $(50 \mathrm{mM}$ TRIS, 2\% SDS, DTT) at room temperature (RT) and then denatured for 5 minutes at $99^{\circ} \mathrm{C}$. Western blot assay was performed as described by Pawłowicz et al. (2012) (53). Briefly, proteins were separated by 12\% SDS-polyacrylamide gel and electroblotted (Trans-blot SD, Semi-Dry Transfer Cell, Bio-Rad) onto nitrocellulose membranes (Bio-Rad). Immunodetections of pFBA, PGK and GAPDH were performed with the use of polyclonal antibodies (Agrisera) diluted at 1:4000. The protein level of antioxidant enzymes was detected using the commercial rabbit polyclonal antibodies (Agrisera). The GR, GPX, Fe-SOD, Cu/ZnSOD, MnSOD, and CAT antibodies were diluted at 1:4000, whereas the APX antibody was diluted at 1:2000. The membranes were incubated with the antibodies for $1 \mathrm{~h}$. Antigen-antibody complexes were detected using a secondary anti-rabbit IgG-horse radish peroxidase conjugate (Sigma) in dilution 1:20000 (1 h of incubation), chemiluminescent substrates Westar Supernova (Cyanogen) and ChemiDoc ${ }^{\mathrm{TM}}$ Touch Igmagin System (Bio-Rad) to visualize the results.

\section{Chloroplast aldolase activity under drought and recovery}

The activity of pFBA in the leaves was measured according to the modified method of Sibley-Lehninger $(54,55)$. Chloroplast proteins were extracted as described by Kosmala et al. (2012) and Perlikowski et al. (2016) with slight modifications $(5,8)$. The amount of $1 \mathrm{~g}$ of frozen material was ground in a liquid nitrogen and then suspended in $10 \mathrm{ml}$ of chloroplast isolation buffer (CIB) (Sigma) with 0,1\% BSA. The homogenised samples were filtered through a Sefar nitrex filter and centrifuged at $200 \mathrm{~g}$ at RT for 3 min. The collected supernatant was subsequently centrifuged for $15 \mathrm{~min}$ at $900 \mathrm{~g}$ at RT and then washed two times in $4 \mathrm{ml}$ of ClB solution. Each time, the suspension was centrifuged at $900 \mathrm{~g}$ at RT for $15 \mathrm{~min}$. The chloroplast pellet was dissolved in $500 \mu \mathrm{l}$ of $0.1 \mathrm{M}$ phosphate buffer $\left(0.1 \mathrm{M} \mathrm{Na}_{2} \mathrm{HPO}_{4}\right)$ with $3 \%$ Triton $\mathrm{X} 100$, shaken by vortex and centrifuged at $21500 \mathrm{~g}$ in RT for $10 \mathrm{~min}$. The collected supernatant was used to determine the pFBA activity. The volume of $100 \mu \mathrm{l} 0.06 \mathrm{M}$ fructose-1,6-bisphosphate and $140 \mu \mathrm{l}$ of incubation buffer ( $0.05 \mathrm{M}$ 2,4,6-trimethylpyridine, $0.08 \mathrm{M}$ hydrazine sulfate, $0.3 \mathrm{mM}$ sodium iodoacetate) 
$\mathrm{pH} 7.4$ was pre-incubated in water bath for $10 \mathrm{~min}$ at $30^{\circ} \mathrm{C}$. Additionally, a material sample was performed for each biological replication which contained $100 \mu$ of $0.06 \mathrm{M}$ fructose-1,6-bisphosphate, $140 \mu$ l of incubation buffer and $300 \mu$ of $10 \%$ trichloroacetic acid (TCA). The volume of $20 \mu$ of chloroplast extract was added to the pre-incubated solutions, mixed and incubated at $30^{\circ} \mathrm{C}$ for $45 \mathrm{~min}$. The reaction was stopped by adding $300 \mu \mathrm{l}$ of $10 \%$ TCA to the solution, and tubes were chilled on ice. Icechilled samples were centrifuged at $21500 \mathrm{~g}$ for $10 \mathrm{~min}$ in RT to remove the precipitated proteins. The volume of $100 \mu$ l of each supernatant was incubated with $100 \mu l$ of $0.75 \mathrm{M} \mathrm{NaOH}$ at RT for 10 min and after that $100 \mu$ of $0.1 \% 2,4$-dinitrophenylhydrasine was added and the samples were incubated for 10 minutes in a water bath at $30^{\circ} \mathrm{C}$. The tubes were taken out and $700 \mu \mathrm{l}$ of $0.75 \mathrm{M} \mathrm{NaOH}$ was added to them and mixed well. After $3 \mathrm{~min}$ of incubation, the absorbance at $540 \mathrm{~nm}$ was measured against the material sample. A standard curve was prepared with use of $0.01 \mathrm{mM}$ D-glyceraldehyde as described in Perlikowski et al. (2016) (8). The amount of produced trioses in the pFBA assay was read according to the standard curve and after calculations of glyceraldehyde $\mathrm{mg}$ produced by $1 \mathrm{~g}$ of plant sample during 1 h.

\section{Lipid peroxidation under drought and recovery (TBARS assay)}

The level of lipid peroxidation was measured spectrophotometrically as a content in the samples of the thiobarbituric-reactive substances (TBARS) according to the method of Heath and Packer (1968) with slight modifications $(56,57,58)$. Briefly, $300 \mathrm{mg}$ of fresh leaves were homogenized with $2 \mathrm{ml}$ of a buffer containing $0.25 \%$ TBA in $10 \%$ TCA at RT. After homogenization, samples were incubated at $100{ }^{\circ} \mathrm{C}$ for 15 min in a water bath. Next, the samples were cooled on ice and centrifuged at $10000 \mathrm{~g}$ by $10 \mathrm{~min}$ at $4{ }^{\circ} \mathrm{C}$. The supernatant was collected and the absorbance was measured at $\lambda=532 \mathrm{~nm}$ and at $\lambda=600$. Amount of TBARS was calculated through the following formula: TBARS $(\mu M)=\left(A_{532}-A_{600}\right) / 155$, where 155 was an extinction factor.

\section{Superoxide anion radical and hydrogen peroxide under drought and recovery}

The level of superoxide anion radical $\left(\mathrm{O}_{2}^{--}\right)$and hydrogen peroxide $\left(\mathrm{H}_{2} \mathrm{O}_{2}\right)$, were assayed spectrophotometrically. Superoxide anion radical measurement was performed according to Doke (1983), and Arasimowicz et al. $(2009)(59,60)$. Nitroblue tetrazolium (NBT) was used as a substrate which undergoes reduction by $\mathrm{O}_{2}{ }^{--}$to form diformazan. Leaf discs $(0.6-0.8 \mathrm{~cm}$ in diameter) were incubated with $3 \mathrm{ml}$ of mixture containing $0.05 \mathrm{M}$ potassium-phosphate buffer ( $\mathrm{pH} 7.8$ ) with $0.1 \mathrm{mM}$ EDTA, $10 \mathrm{mM} \mathrm{NaN}_{3}$ and $0.05 \% \mathrm{NBT}$, for $1 \mathrm{~h}$ in the dark. Next, the samples were heated at $85^{\circ} \mathrm{C}$ by $15 \mathrm{~min}$ and cooled down on ice. The absorbance was measured at $\lambda=580 \mathrm{~nm}$. The level of $\mathrm{O}_{2}{ }^{-{ }^{-}}$was expressed as absorbance at 580 $\mathrm{nm}$ per $1 \mathrm{~g}$ of fresh weight (FW). 
The concentration of hydrogen peroxide $\left(\mathrm{H}_{2} \mathrm{O}_{2}\right)$ was assayed using the titanium $\left(\mathrm{Ti}^{+}\right)$method $(61,62)$. The amount of $400 \mathrm{mg}$ of plant tissue was homogenized on ice with $1.5 \mathrm{ml}$ of $0.1 \mathrm{M}$ potassiumphosphate buffer, $\mathrm{pH} 7.8$. Obtained extracts were centrifuged at $14000 \mathrm{~g}$ at $4{ }^{\circ} \mathrm{C}$ by $25 \mathrm{~min}$. The volume of $1.5 \mathrm{ml}$ of reaction mixture containing $400 \mu \mathrm{l}$ of enzymatic extract, $600 \mu \mathrm{l}$ of potassium-phosphate buffer and $500 \mu$ l of titanium reagent ( $0.6 \mathrm{mM}$ PRL and $0.6 \mathrm{mM}$ PTO in a ratio 1:1) were prepared for each samples. After 10 min of incubation, the absorbance was measured at $\lambda=508 \mathrm{~nm}$. The standard curve was used. The level of $\mathrm{H}_{2} \mathrm{O}_{2}$ was determined on the basis of absorbance and expressed as $\mu \mathrm{mol}$ $\mathrm{H}_{2} \mathrm{O}_{2}$ per $1 \mathrm{~g}$ of fresh weight (FW).

\section{Statistical analysis}

All the statistical analyses were performed with the STATISTICA 10.0 software (StatSoft, Tulsa OK, USA). A two-way analyses of variance (ANOVA), with genotype and time-point as classification factors, were performed. Differences in physiological parameters, protein accumulation, pFBA activity, RT-qPCR, TBARS assay, ROS measurement between the plants during experiment duration were evaluated using Fisher's least significant difference (LSD) test at $P=0.01$. Homogeneity groups according to test were denoted by the same letters on the graphs.

\section{Abbreviations}

ABS/CS energy flux for absorbed energy/CS

$A B S / R C \quad$ energy flux for absorbed energy/RC

APX L-ascorbate peroxidase

BSA bovine serum albumin

CAT catalase

$\mathrm{Ci} \quad$ internal $\mathrm{CO} 2$ concentration

CO2 carbon dioxide

CS cross section of the leaf tissue

$\mathrm{Cu} / \mathrm{Zn}$-SOD chloroplastic $\mathrm{Cu} / \mathrm{Zn}$ superoxide dismutase

DI0/CS energy flux for dissipated energy/CS

DI0/RC energy flux for dissipated energy/RC

DTT DL-dithiothreitol 


\begin{tabular}{|c|c|}
\hline DW & leaf dry weight \\
\hline EDTA & ethylenediaminetetraacetic acid \\
\hline EL & electrolyte leakage \\
\hline ETO/CS & electron transport flux per CS \\
\hline Fe-SOD & Fe-dependent superoxide dismutase \\
\hline FISH & Fluorescent in situ hybridization \\
\hline FW & Fe-dependent superoxide dismutase \\
\hline GAPDH & glyceraldehyde-3-phosphate dehydrogenase \\
\hline gs & stomatal conductance \\
\hline GPX & glutathione peroxidase \\
\hline GR & glutathione reductase \\
\hline HDR & high drought tolerant \\
\hline L1 & electrical conductivity of the fresh leaf \\
\hline L2 & electrical conductivity of the leaf immersed in liquid nitrogen \\
\hline LDR & low drought tolerant (LDR) \\
\hline Mn-SOD & manganese superoxide dismutase \\
\hline SDS & sodium dodecyl sulfate \\
\hline SOD & superoxide dismutase \\
\hline pFBA & fructose-1,6-bisphosphate aldolase \\
\hline PGK & phosphoglycerate kinase \\
\hline PPFD & photosynthetic photon flux density \\
\hline PRL & 4-(2'-pyridylazo)-resorcinol \\
\hline PSII & photosystem II \\
\hline PTO & potassium sodium tartrate tetrahydrate \\
\hline
\end{tabular}


$\mathrm{RC} / \mathrm{CSO} \quad$ densities of active PSIl at $\mathrm{t}=0 / \mathrm{CS}$

$\mathrm{RC} / \mathrm{CSm}$ densities of active PSII reaction centers at tmax (time to reach maximum fluorescence)

ROS reactive oxygen species

RWC relative water content

TBARS the thiobarbituric-reactive substances

TRO/CS energy flux for trapped energy/CS

TRO/RC energy flux for trapped energy/RC

\section{Supplementary Information}

Additional files 1: Figure S1. Chlorophyll fluorescence measurments: ABS/RC (A), ABS/CS (B), TRO/RC (C), TRO/CS (D) and ETO/CS (E) in two genotypes of $F$. arundinacea (Fa-HDR, Fa-LDR) and $F$. glaucescens (Fg-HDR, Fg-LDR) before stress treatment (C), on the $3^{\text {rd }}(D 1), 6^{\text {th }}$ (D2) and $11^{\text {th }}$ (D3) day of water deficit and 10 days after re-hydration initiation $(\mathrm{RH})$. The data represent means for ten individual measurements, error bars represent the standard errors (SE). Homogeneity groups according to Fischer LSD test (P = 0.01) are denoted by the same letters.

Additional files 2: Figure S2. Chlorophyll fluorescence measurments: RC/CSm (A), RC/CSO (B), DI0/RC (C), DI0/CS (D) in two genotypes of $F$. arundinacea (Fa-HDR, Fa-LDR) and F. glaucescens (Fg-HDR, FgLDR) before stress treatment (C), on the $3^{\text {rd }}$ (D1), $6^{\text {th }}$ (D2) and $11^{\text {th }}$ (D3) day of water deficit and 10 days after re-hydration initiation (RH). The data represent means for ten individual measurements, error bars represent the standard errors (SE). Homogeneity groups according to Fischer LSD test $(P=0.01)$ are denoted by the same letters.

\section{Declarations}

\section{Ethics approval and consent to participate}

Not applicable.

\section{Consent for publication}

Not applicable. 


\section{Availability of data and materials}

Not applicable.

\section{Competing interests}

The authors declare that they have no competing interests.

\section{Funding:}

The research was supported by the National Science Centre (project no. 2016/23/B/NZ9/00820). Funding bodies were not involved in the design of the study and collection, analysis, interpretation of data and in writing the manuscript.

\section{Authors' contributions}

AK, MA-J conceived the ideas and designed the experiment. KL, IP, DP, AA and JM performed the experiments. $K L$ performed the data analysis, $K L$ and $I P$ made a data interpretation. $K L$ and JM prepared the figures and table. DP made statistics analysis. KL and IP wrote the article. AK and MA-J checked the manuscript.

\section{Acknowledgements}

We thank the Institute of Biological, Environmental and Rural Sciences (IBERS) (UK) for the seeds of Festuca glaucescens.

\section{References}

1. Gray SB, Brady SM. Plant developmental responses to climate change. Developmental Biology. 2016;419:64-7765.

2. Marshall AH, Collins RP, Humphreys MW, Scullion J. A new emphasis on root traits for perennial grass and legume varieties with environmental and ecological benefits. Food Energy Secur. 2016;5(1):26-39.

3. Augustyniak A, Perlikowski D, Rapacz M, Kościelniak J, Kosmala A. Insight into cellular proteome of Lolium multiflorum/Festuca arundinacea introgression forms to decipher crucial mechanisms of cold acclimation in forage grasses. Plant Sci. 2018;272:22-31.

4. Kosmala A, Bocian A, Rapacz M, Jurczyk B, Zwierzykowski Z. Identification of leaf proteins differentially accumulated during cold acclimation between Festuca pratensis plants with distinct levels of frost tolerance. J Exp Bot. 2009;60:3595- 
5. Kosmala A, Perlikowski D, Pawłowicz I, Rapacz M. Changes in the chloroplast proteome following water deficit and subsequent watering in a high and a low drought tolerant genotype of Festuca arundinacea. J Exp Bot. 2012;63:6161-

6. Pawłowicz I, Rapacz M, Perlikowski D, Gondek K, Kosmala A. Abiotic stresses influence the transcript abundance of PIP and TIP aquaporins in Festuca J Appl Genet. 2017;58(4):421-435.

7. Pawłowicz I, Waśkiewicz A, Perlikowski D, Rapacz M, Ratajczak D, Kosmala A. Remodeling of chloroplast proteome under salinity affects salt tolerance of Festuca arundinacea. Photosynth Res. 2018;137:475-

8. Perlikowski D, Czyżniejewski M, Marczak $Ł$, Augustyniak A, Kosmala A. Water deficit affects primary metabolism differently in two Lolium multiflorum/Festuca arundinacea introgression forms with a distinct capacity for photosynthesis and membrane regeneration. Front Plant Sci. 2016;7:1063.

9. Perlikowski D, Augustyniak A, Masajada K, Skirycz A, Soja AM, Michaelis Ä, Wolter G, Kosmala A. Structural and metabolic alterations in root systems under limited water conditions in forage grasses of Lolium-Festuca complex. Plant Sci. 2019;283:211-223.

10. Humphreys MW, Thomas HM, Morgan WG, Meredith MR, Harper JA, Thomas H, Zwierzykowski Z, Ghesquière M. Discriminating the ancestral progenitors of hexaploid Festuca arundinacea using genomic in situ Heredity. 1995;75:171-174.

11. Thomas $\mathrm{H}$, Humphreys MO. Progress and potential of interspecific hybrids of Lolium and Festuca. $\mathrm{J}$ Agric Sci. 1991;117:1-8.

12. Humphreys MW, Thomas HM, Harper J, Morgan G, James A, Ghamari-Zare A, Thomas H. Dissecting drought-and cold-tolerance traits in the Lolium-Festuca complex by introgression mapping. New Phytol. 1997;137:55-

13. Humphreys J, Harper JA, Armstead IP, Humphreys MW. Introgression-mapping of genes for drought resistance transferred from Festuca arundinacea glaucescens into Lolium multiflorum. Theor Appl Genet. 2005;110:579-587.

14. Fang $Y$, Xiong $L$. General mechanisms of drought response and their application in drought resistance improvement in plants. Cell Mol Life Sci. 2015;72:673-689.

15. Reddy AR, Chaitanya KV, Vivekanandan M. Drought-induced responses of photosynthesis and antioxidant metabolism in higher plants. J Plant Physiol. 2004;161:1189-1202.

16. Perlikowski D, Kosmala A, Rapacz M, Kościelniak J, Pawłowicz I, Zwierzykowski Z. Influence of shortterm drought conditions and subsequent re-watering on the physiology and proteome of Lolium multiflorum/Festuca arundinacea introgression forms with contrasting levels of tolerance to longterm drought. Plant Biol. 2014;16:385-394.

17. Gill SS, Tuteja N. Reactive oxygen species and antioxidant machinery in abiotic stress tolerance in crop plants. Plant Physil Bioch. 2010;48:909-

18. Hossain MA, Bhattacharjee S, Armin SM, Qian P, Xin W, Li HY, Tran LSP. Hydrogen peroxide priming modulates abiotic oxidative stress tolerance: insights from ROS detoxification and scavenging. Front Plant Sci. 2015;6:420. 
19. Miller G, Suzuki N, Ciftci-Yilmaz S, Mittler R. Reactive oxygen species homeostasis and signalling during drought and salinity stresses. Plan Cell and Environ. 2010;33:453-467.

20. Baxter A, Mittler R, Suzuki N. ROS as key players in plant stress signaling. J Exp Bot. 2014;65:12291240.

21. You J, Chan Z. ROS Regulation during abiotic stress responses in crop plants. Front Plant Sci. 2015;6:092.

22. Sofo A, Dichio B, Xiloyannis C, Masia A. Effects of different irradiance levels on some antioxidant enzymes and on malondialdehyde content during rewatering in olive tree. Plant Sci. 2004;166:293-

23. Campo S, Baldrich P, Messeguer J, Lalanne E, Coca M, San Segundo B. Overexpression of a calciumdependent protein kinase confers salt and drought tolerance in rice by preventing membrane lipid peroxidation. Plant Physiol. 2014;165:688-704.

24. Gharibi S, Tabatabaei BE; Saeidi SG, Goli SAH. Effect of drought stress on total phenolic, lipid peroxidation, and antioxidant activity of Achillea Appl Biochem Biotech. 2016;178:796-809.

25. Cruz de Carvalho MH. Drought stress and reactive oxygen species: Production, scavenging and signalling. Plant Signal Behav. 2008;3:156-165.

26. Dubey AK, Kumar N, Kumar A, Ansari MA, Ranjan R, Gautam A, Meenakshi, Sahu N, Pandey V, Behera SK, Mallick S, Pande V, Sanyal I. Over-expression of CarMT gene modulates the physiological performance and antioxidant defense system to provide tolerance against drought stress in Arabidopsis thaliana Ecotoxicol Environ Saf. 2019;171:54-65.

27. Iqbal N, Hussain S, Raza MA, Yang CQ, Safdar ME, Brestic M, Aziz A, Hayyat MS, Asghar MA, Wang XC, Zhang J, Yang W, Liu J. Drought Tolerance of Soybean (Glycine max Merr.) by Improved Photosynthetic Characteristics and an Efficient Antioxidant Enzyme Activities Under a Split-Root System. Front Physiol. 2019;10:786.

28. Mittler R, Vanderauwera S, Gollery M, Van Breusegem F. Reactive oxygen gene network of plants.Trends Plant Sci. 2004;9:490-

29. Bailey-Serres $\mathrm{J}$, Mittler R. The roles of reactive oxygen species in plant cells. Plant Physiol. 2006;141(2):311.

30. Laxa M, Liebthal M, Telman W, Chibani K, Dietz K-J. The Role of the Plant Antioxidant System in Drought Tolerance. Antioxidants. 2019;8:94.

31. Zhang C, Shi S, Liu Z, Yang F, Yin G. Drought tolerance in alfalfa (Medicago sativa) varieties is associated with enhanced antioxidative protection and declined lipid peroxidation. J Plant Physiol. 2019;232:226-240.

32. Luna CM, Pastori GM, Driscoll S, Groten K, Bernard S, Foyer CH. Drought controls on $\mathrm{H}_{2} \mathrm{O}_{2}$ accumulation, catalase (CAT) activity and CAT gene expression in wheat. J Exp Bot. 2005;56:417423.

33. Perlikowski D, Augustyniak A, Skirycz A, Pawłowicz I, Masajada K, Michaelis Ä, Wolter G, Kosmala A. Efficient root metabolism improves drought resistance of Festuca arundinacea. Plant Cell Physiol. 
2019;in print. DOI: 10.1093/pcp/pcz215.

34. Thomas HM, Harper JA, Meredith MR, Morgan WG, King IP. Physical mapping of ribosomal DNA sites in Festuca arundinacea and related species by in situ Genome. 1997;40:406-410.

35. Rampino P, Pataleo S, Gerardi C, Mita, G, Perrotta C. Drought stress response in wheat: physiological and molecular analysis of resistant and sensitive genotypes. Plant Cell Environ. 2006;29:2143-2152.

36. Wasson AP, Richards RA, Chatrath R, Misra SC, Sai Prasad SV, Rebetzke GJ, Kirkegaard JA, Christopher J, Watt M. Traits and selection strategies to improve root systems and water uptake in water-limited wheat crops. J Exp Bot. 2012;63(9)3485-3498.

37. Maxwell K, Johnson GN. Chlorophyll fluorescence - a practical guide. J Exp Bot. 2000;51:659-668.

38. Zandalinas SI, Balfagón D, Arbona V, Gómez-Cadenas A, Inupakutika MA, Mittler R. ABA is required for the accumulation of APX1 and MBF1c during a combination of water deficit and heat stress. $J$ Exp Bot. 2016;67:5381-5390.

39. Zhao F, Zhang D, Zhao Y, Wang W, Yang H, Tai F, Li C, Hu X. The difference of physiological and proteomic changes in maize leaves adaptation to drought, heat, and combined both stresses. Front Plant Sci. 2016;7:1471.

40. Jin R, Wang Y, Liu R, Gou J, Chan Z. Physiological and metabolic changes of purslane (Portulaca oleracea) in response to drought, heat, and combined stresses. Front Plant Sci. 2016;6:1123.

41. Lawlor DW, Cornic G. Photosynthetic carbon assimilation and associated metabolism in relation to water deficits in higher plants. Plant Cell Environ. 2002;25:275-294.

42. Choudhury FK, Rivero RM, Blumwald E, Mittler R. Reactive oxygen species, abiotic stress and stress combination. Plant J. 2017;90:856-867.

43. Avramova V, AbdElgawad H, Vasileva I, Petrova AS, Holek A, Mariën J, Asard H, Beemster GTS. High Antioxidant Activity Facilitates Maintenance of Cell Division in Leaves of Drought Tolerant Maize Hybrids. Front Plant Sci. 2017;8:84.

44. Sheoran S, Thakur V, Narwal S, Turan R, Mamrutha HM, Singh V, Tiwari V, Sharma I. Differential Activity and Expression Profile of Antioxidant Enzymes and Physiological Changes in Wheat (Triticum aestivum) Under Drought. Appl Biochem Biotech. 2015;177(6):1282-1298.

45. Harb A, Awad D, Samarah N. Gene expression and activity of antioxidant enzymes in barley (Hordeum vulgare) under controlled severe drought. Plant-Environment Interactions. 2015;10(1):109116.

46. Alscher RG, Erturk N, Heath LS. Role of superoxide dismutases (SODs) in controlling oxidative stress in plants. J Exp Bot. 2002;53(372):1331-1341.

47. Mhamdi A, Queval G, Chaouch S, Vanderauwera S, Van Breusegem F, Noctor G, Catalase function in plants: a focus on Arabidopsis mutants as stress-mimic models. J Exp Bot. 2010;61(15):4197-4220.

48. Alché JD. A concise appraisal of lipid oxidation and lipoxidation in higher plants. Redox Biol. 2019;7:101136. 
49. Xie X, He Z, Chen N, Tang Z, Wang Q, Cai Y. The Roles of Environmental Factors in Regulation of Oxidative Stress in Plant. Biomed Res Int. 2019;9732325.

50. Majka J, Książczyk T, Kiełbowicz-Matuk A, Kopecký D, Kosmala A. Exploiting repetitive sequences and BAC clones in Festuca pratensis PLOS ONE. 2017;12(6):e0179043.

51. Hurkman WJ, Tanaka CK. Solubilization of plant membrane proteins for analysis by two-dimensional gel electrophoresis. Plant Physiol. 1986;81:802-806.

52. Masojć P, Kosmala A, Perlikowski D. Proteomic analysis of developing rye grain with contrasting resistance to preharvest sprouting. J Appl Genetics. 2013;54:11-19.

53. Pawłowicz I, Kosmala A, Rapacz M. Expression pattern of the psbO gene and its involvement in acclimation of the photosynthetic apparatus during abiotic stresses in Festuca arundinacea and pratensis. Acta Physiol Plant. 2012;34:1915-1924.

54. Sibley JA, Lehninger AL. Determination of aldolase in animal tissues. J Biol Chem. 1949;177:859872.

55. Willard JM, Gibbs M. Role of aldolase in photosynthesis. II demonstration of aldolase types in photosynthetic organisms. Plant Physiol. 1968;43:793-798.

56. Heath RL, Packer L. Photoperoxidation in isolated chloroplasts. I. Kinetics and stoichiometry of fatty acid peroxidation. Arch Biochem Biophys. 1968;125:180-198.

57. Yagi K. Assay for serum lipid peroxide level its clinical significance. In: Yagi K, editors. Lipid peroxides in biology and medicine. London, New York: Academic Press Inc.; 1982. p. 223-241.

58. Arasimowicz-Jelonek M, Floryszak-Wieczorek J, Kosmala A. Are nitric oxide donors a valuable tool to study the functional role of nitric oxide in plant metabolism? Plant Biol. 2011;13:747-756.

59. Doke N. Involvement of superoxide anion generation in the hypersensitive response of potato tuber tissues to infection with an incompatible race of Phytophthora infestans and to the hyphal wall components. Physiol Plant Pathol. 1983;23:345-

60. Arasimowicz M, Floryszak-Wieczorek J, Milczarek G, Jelonek T. Nitric oxide, induced by wounding, mediates redox regulation in pelargonium leaves. Plant Biol. 2009;11:650-663.

61. Becana M, Aparicio-Tejo P, Irigoyen JJ, Sanchez-Diaz M. Some enzymes of hydrogen peroxide metabolism in leaves and root nodules of Medicago sativa. Plant Physiol. 1986;82:1169-1171.

62. Arasimowicz-Jelonek M, Floryszak-Wieczorek J, Gzyl J, Chmielowska-Bąk J. Homocysteine overaccumulation as the effect of potato leaves exposure to biotic stress. Plant Physiol Bioch. 2013;63:177-184.

\section{Table}

Table 1 Abbreviations of $F$. arundinacea and $F$. glaucescens genotypes used in the analyses and their definitions. 


\begin{tabular}{lll}
\hline F. arundinacea & Fa-HDR & high drought resistant \\
& Fa-LDR & low drought resistant \\
\hline F. glaucescens & Fg-HDR & high drought resistant \\
& Fg-LDR & low drought resistant \\
\hline
\end{tabular}

\section{Figures}




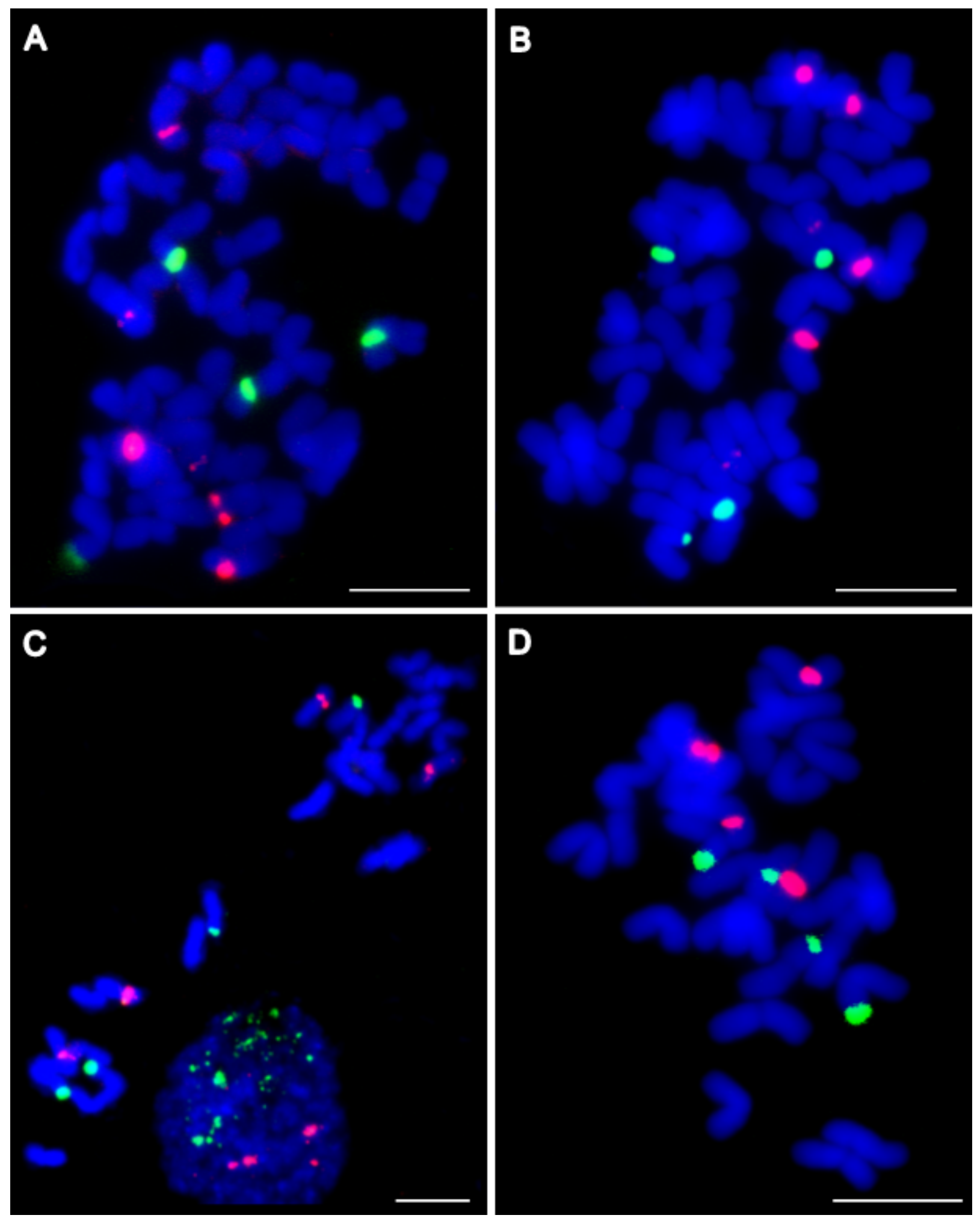

Figure 1

Distribution of rDNA sequences in metaphase chromosomes of $F$. arundinacea $(2 n=6 x=42)$ and $F$. glaucescens $(2 n=4 x=28)$. Fa-HDR genotype of F. arundinacea (A); Fa-LDR genotype of F. arundinacea (B); Fg-HDR genotype of F. glaucescens (C); Fg-LDR genotype of F. glaucescens (D). $5 S$ rDNA - red; $35 S$ rDNA - green; chromosomes counterstained with DAPI - blue. Scale bars $5 \mu \mathrm{m}$. 
Figure 2

F. arundinacea and F. glaucescens in the control conditions, on the 11th day of drought (D3) and after rewatering $(\mathrm{RH})$.
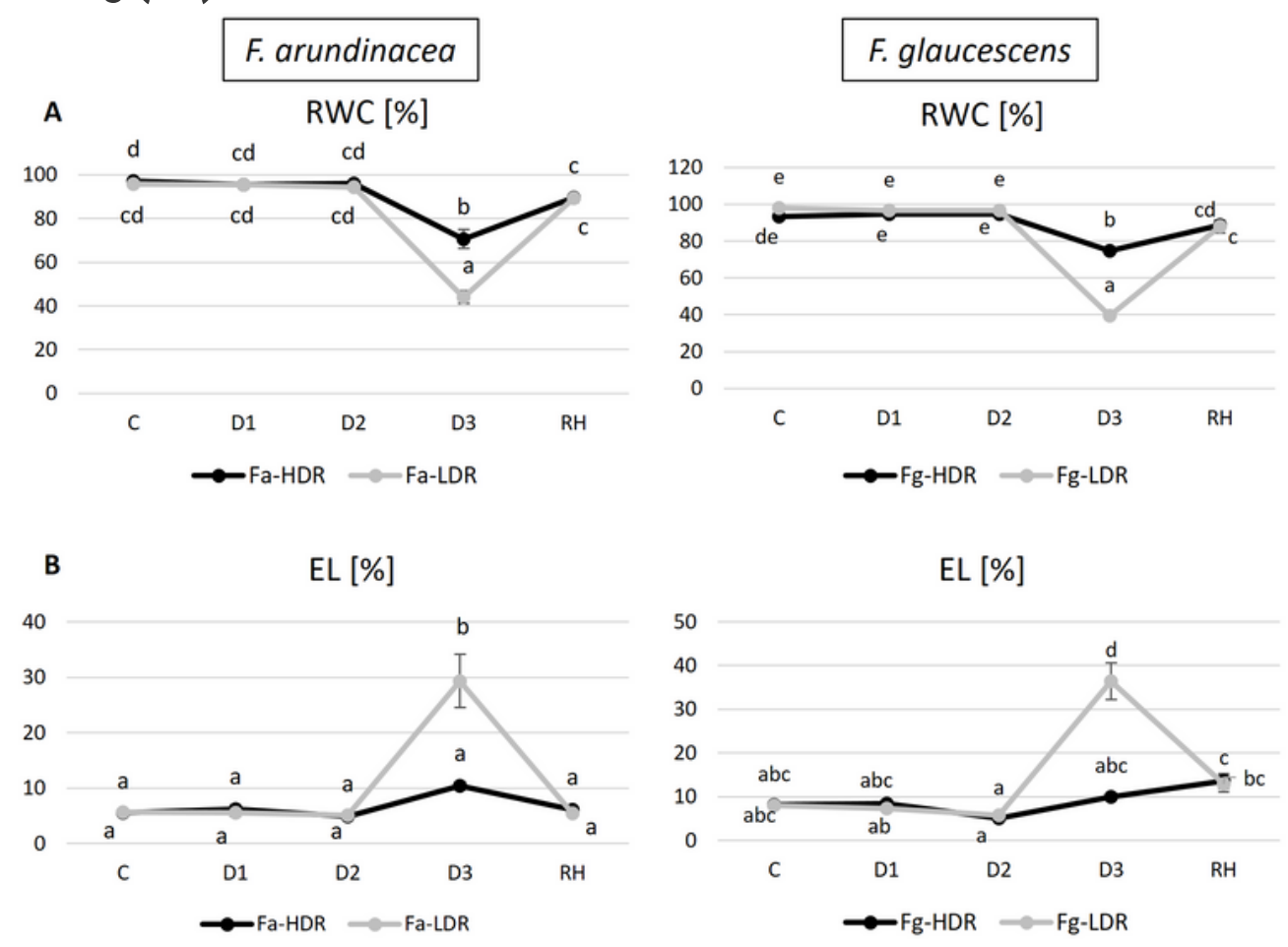

Figure 3 
The relative water content (RWC) (A) and the electrolyte leakage (EL) (B) in two genotypes of F. arundinacea (Fa-HDR, Fa-LDR) and F. glaucescens (Fg-HDR, Fg-LDR) before stress treatment (C), on the 3rd (D1), 6th (D2) and 11th (D3) day of water deficit and 10 days after re-hydration initiation (RH). Error bars represent the standard errors (SE). Homogeneity groups according to Fischer LSD test $(P=0.01)$ are denoted by the same letters.
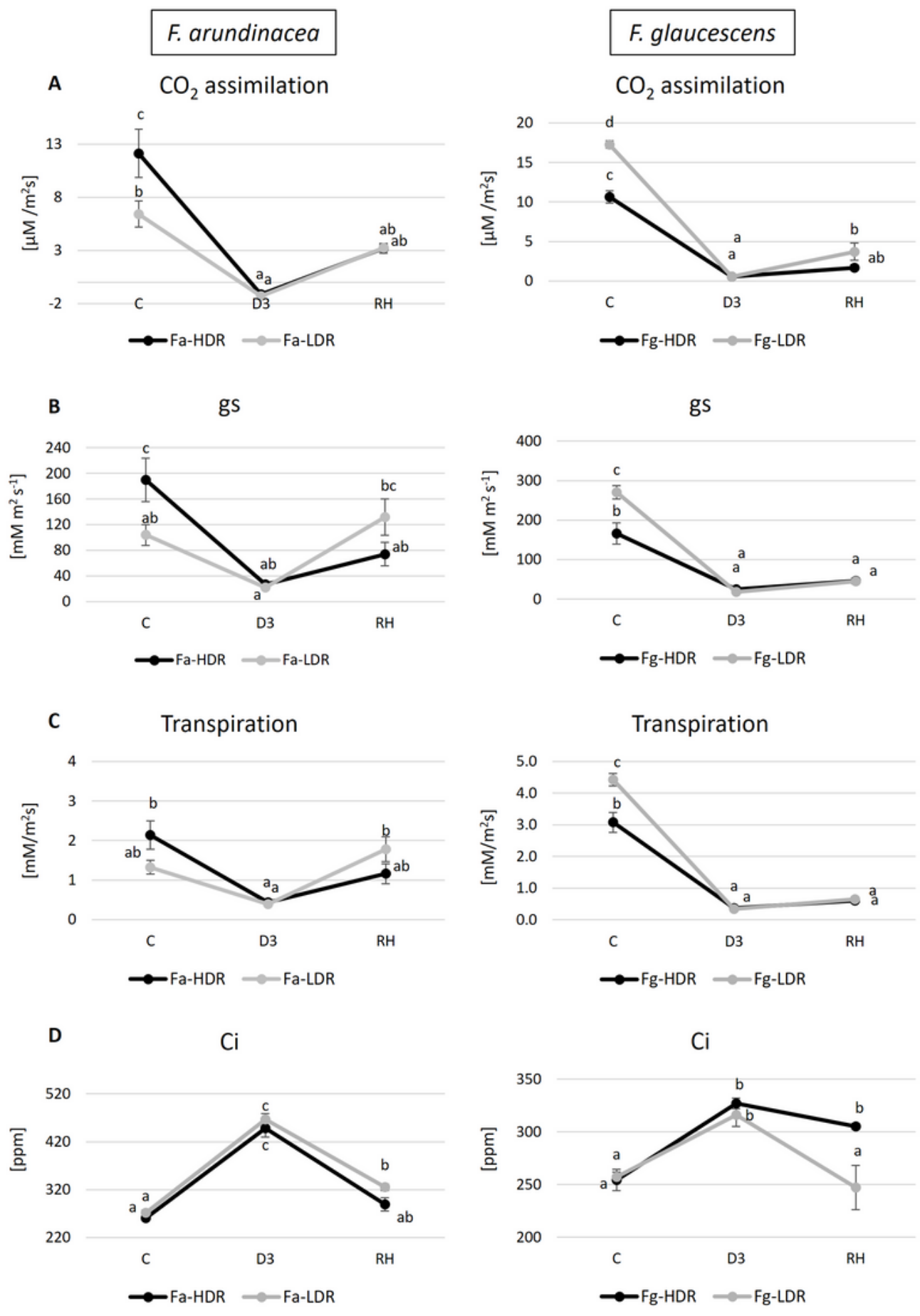

Figure 4 
The gas exchange parameters (CO2 assimilation (A), stomatal conductance (gs) (B), transpiration (C), concentration of $\mathrm{CO} 2(\mathrm{Ci})(\mathrm{D})$ ) in two genotypes of F. arundinacea (Fa-HDR, Fa-LDR) and F. glaucescens (Fg-HDR, Fg-LDR) before stress treatment (C), on 11th (D3) day of water deficit and 10 days after rehydration initiation (RH). Error bars represent the standard errors (SE). Homogeneity groups according to Fischer LSD test $(P=0.01)$ are denoted by the same letters.
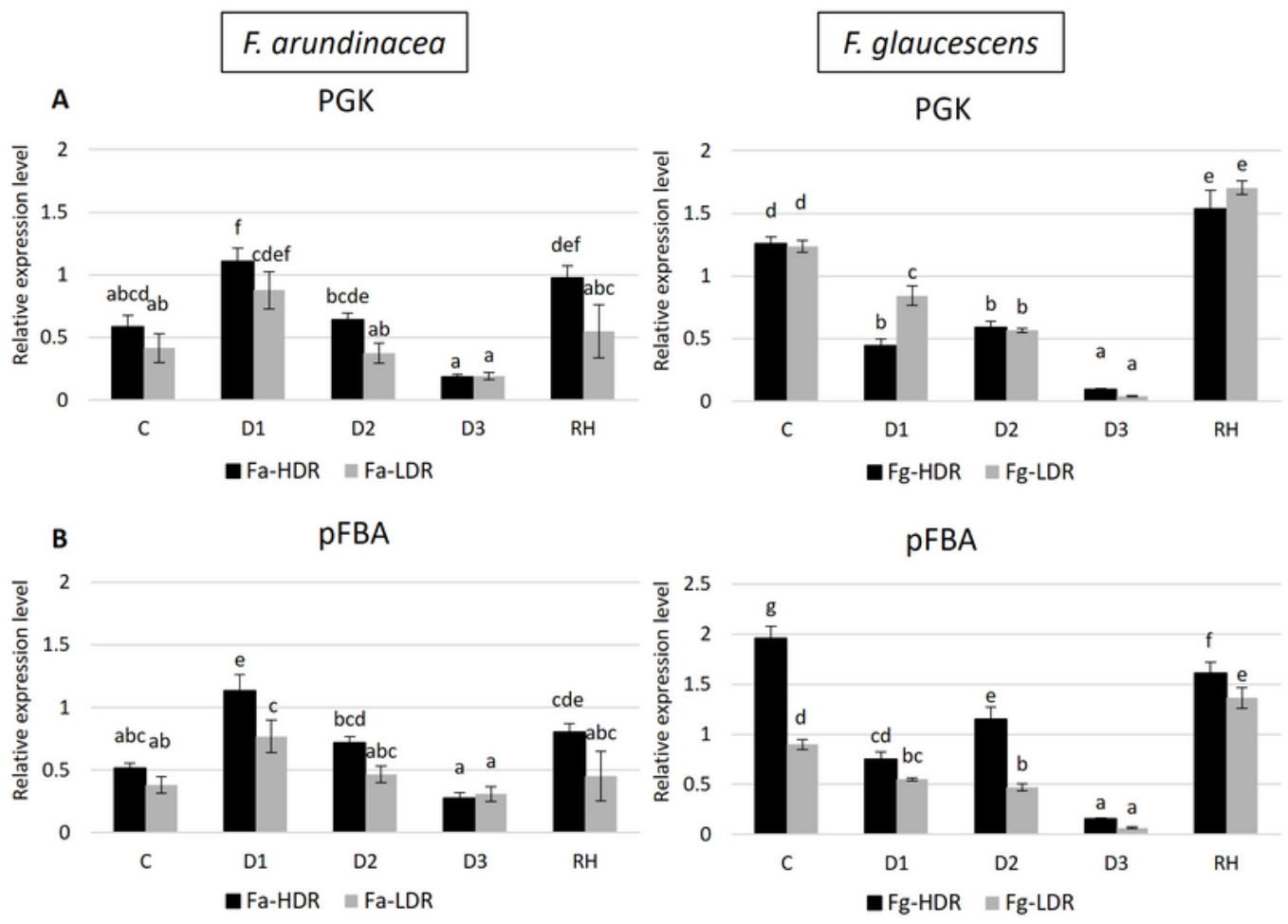

C GAPDH
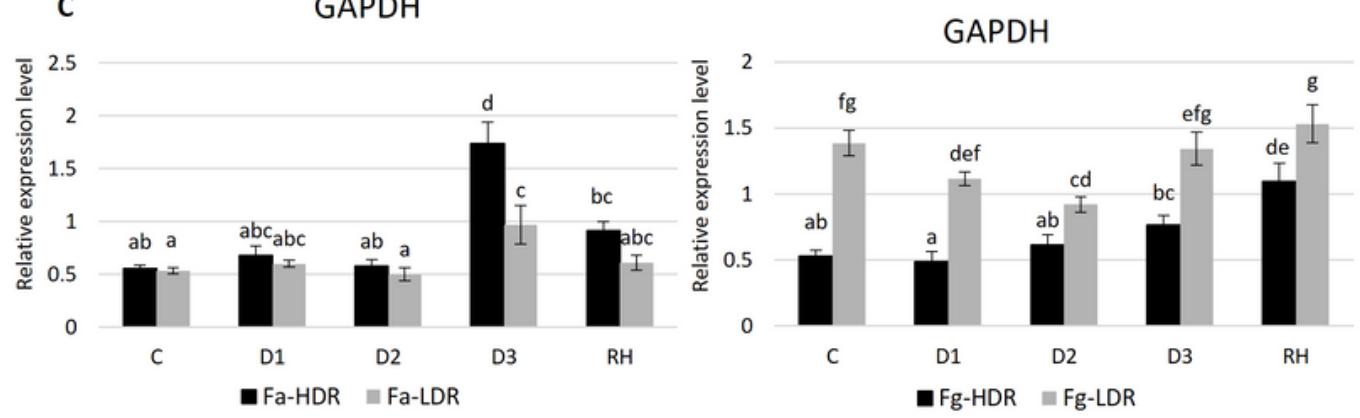

Figure 5 
Relative expression level of phosphoglycerate kinase (PGK) (A), fructose-1,6-bisphosphate aldolase (pFBA) (B), and glyceraldehyde-3-phosphate dehydrogenase (GAPDH) (C) in two genotypes of $F$. arundinacea (Fa-HDR, Fa-LDR) and F. glaucescens (Fg-HDR, Fg-LDR) before stress treatment (C), on the 3rd (D1), 6th (D2) and 11th (D3) day of water deficit and 10 days after subsequent re-watering (RH). The transcript levels of actin and ubiquitin were used as references. Error bars represent the standard errors (SE) of three biological and two technical replicates. Homogeneity groups according to Fischer LSD test $(P=0.01)$ are denoted by the same letters.
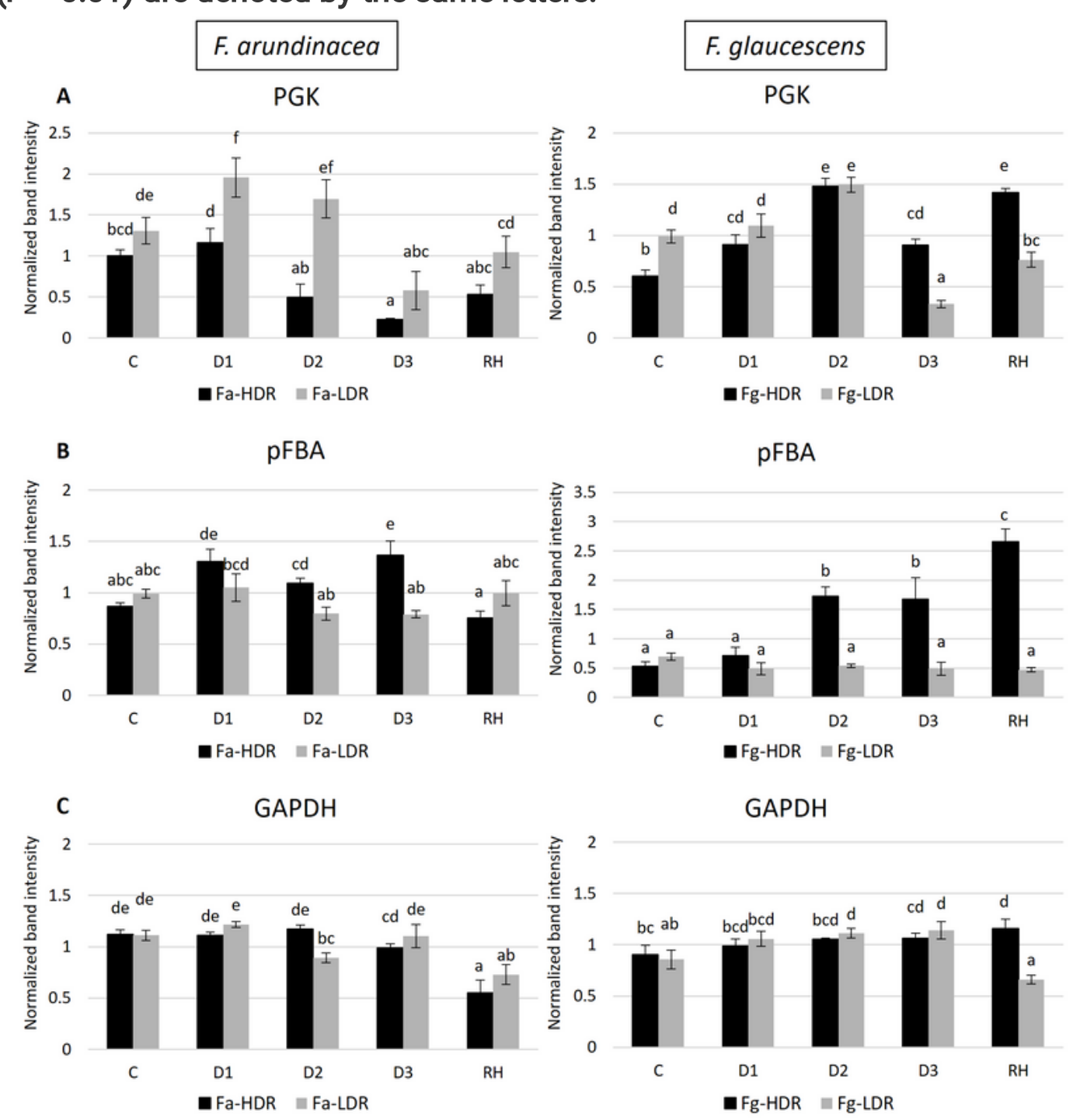
Figure 6

Protein level of phosphoglycerate kinase (PGK) (A), fructose-1,6-bisphosphate aldolase (pFBA) (B), and glyceraldehyde-3-phosphate dehydrogenase (GAPDH) (C) in two genotypes of F. arundinacea (Fa-HDR, Fa-LDR) and F. glaucescens (Fg-HDR, Fg-LDR) before stress treatment (C), on the 3rd (D1), 6th (D2) and 11th (D3) day of water deficit and 10 days after subsequent re-watering (RH). Error bars represent the standard errors (SE) of three biological and two technical replicates. Homogeneity groups according to Fischer LSD test $(P=0.01)$ are denoted by the same letters.

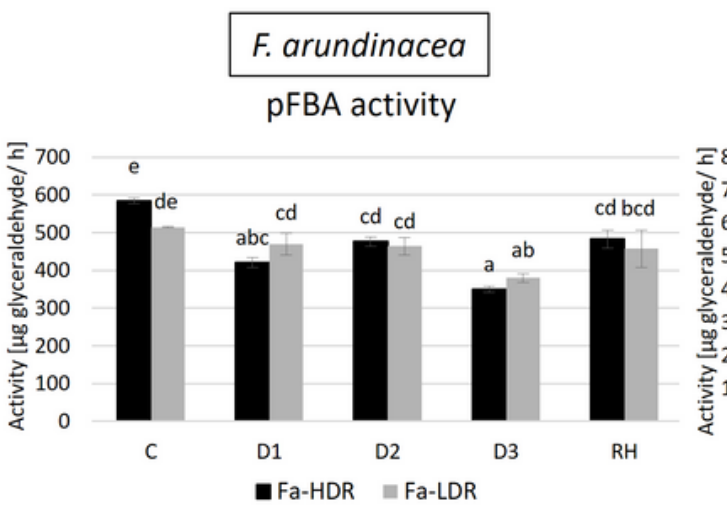

$$
\begin{array}{c|}
\hline \text { F. glaucescens } \\
\hline \text { pFBA activity }
\end{array}
$$

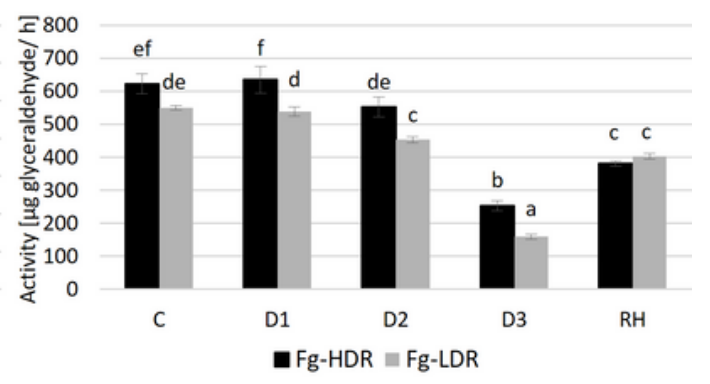


Figure 7

The activity of fructose-1,6-bisphosphate aldolase (pFBA) in two genotypes of F. arundinacea (Fa-HDR, Fa-LDR) and F. glaucescens (Fg-HDR, Fg-LDR) before stress treatment (C), on the 3rd (D1), 6th (D2) and 11th (D3) day of water deficit and 10 days after subsequent re-watering (RH). Error bars represent the standard errors (SE) of three biological and two technical replicates. Homogeneity groups according to Fischer LSD test $(P=0.01)$ are denoted by the same letters.
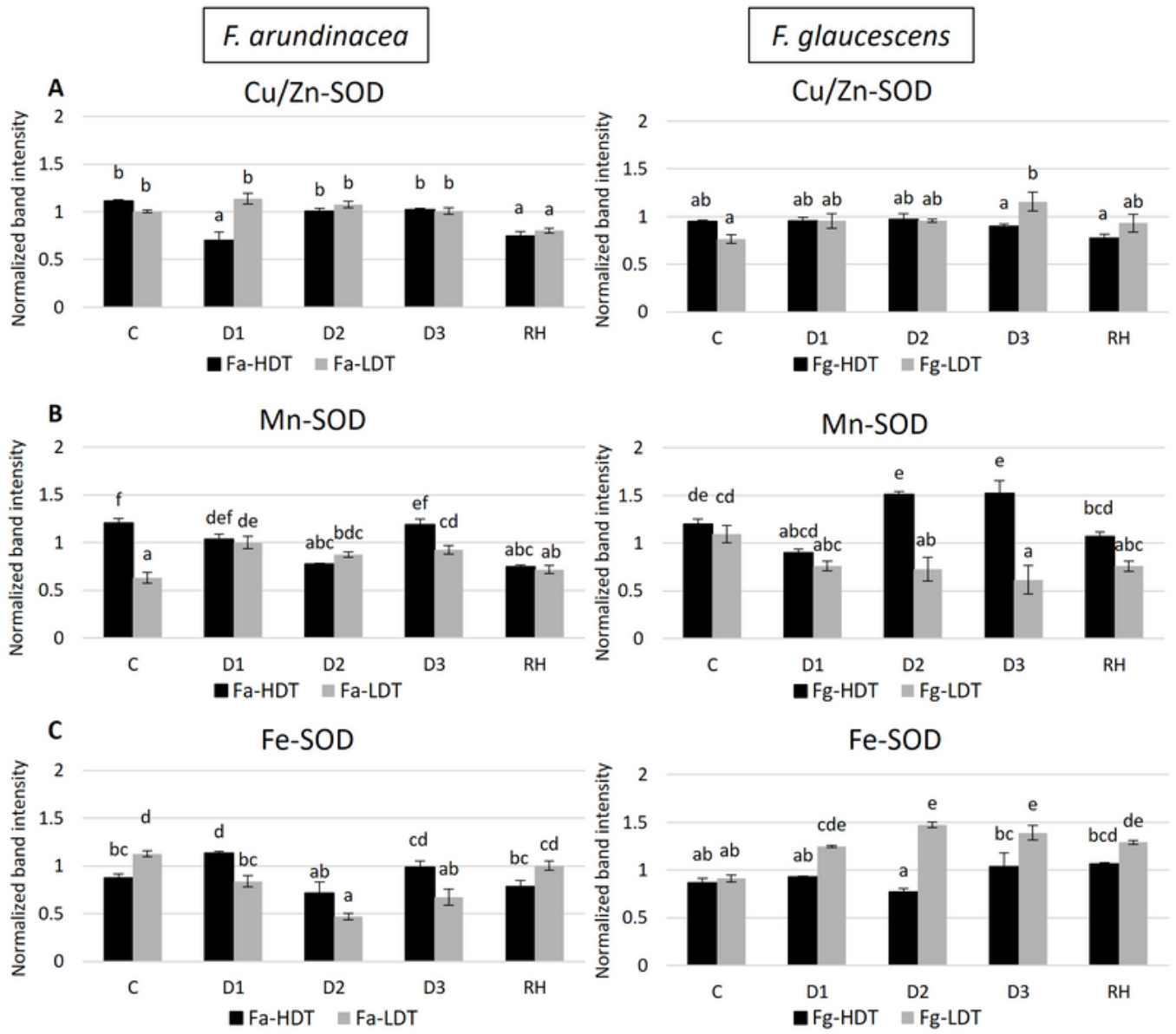
Figure 8

The protein level of $\mathrm{Cu} / \mathrm{Zn}$ superoxide dismutase (Cu/Zn-SOD) (A), manganese superoxide dismutase (Mn-SOD) (B), Fe-dependent superoxide dismutase (Fe-SOD) (C) in two genotypes of F. arundinacea (FaHDR, Fa-LDR) and F. glaucescens (Fg-HDR, Fg-LDR) before stress treatment (C), on the 3rd (D1), 6th (D2) and 11th (D3) day of water deficit and 10 days after subsequent re-watering (RH). Error bars represent the standard errors (SE) of three biological and two technical replicates. Homogeneity groups according to Fischer LSD test $(P=0.01)$ are denoted by the same letters.
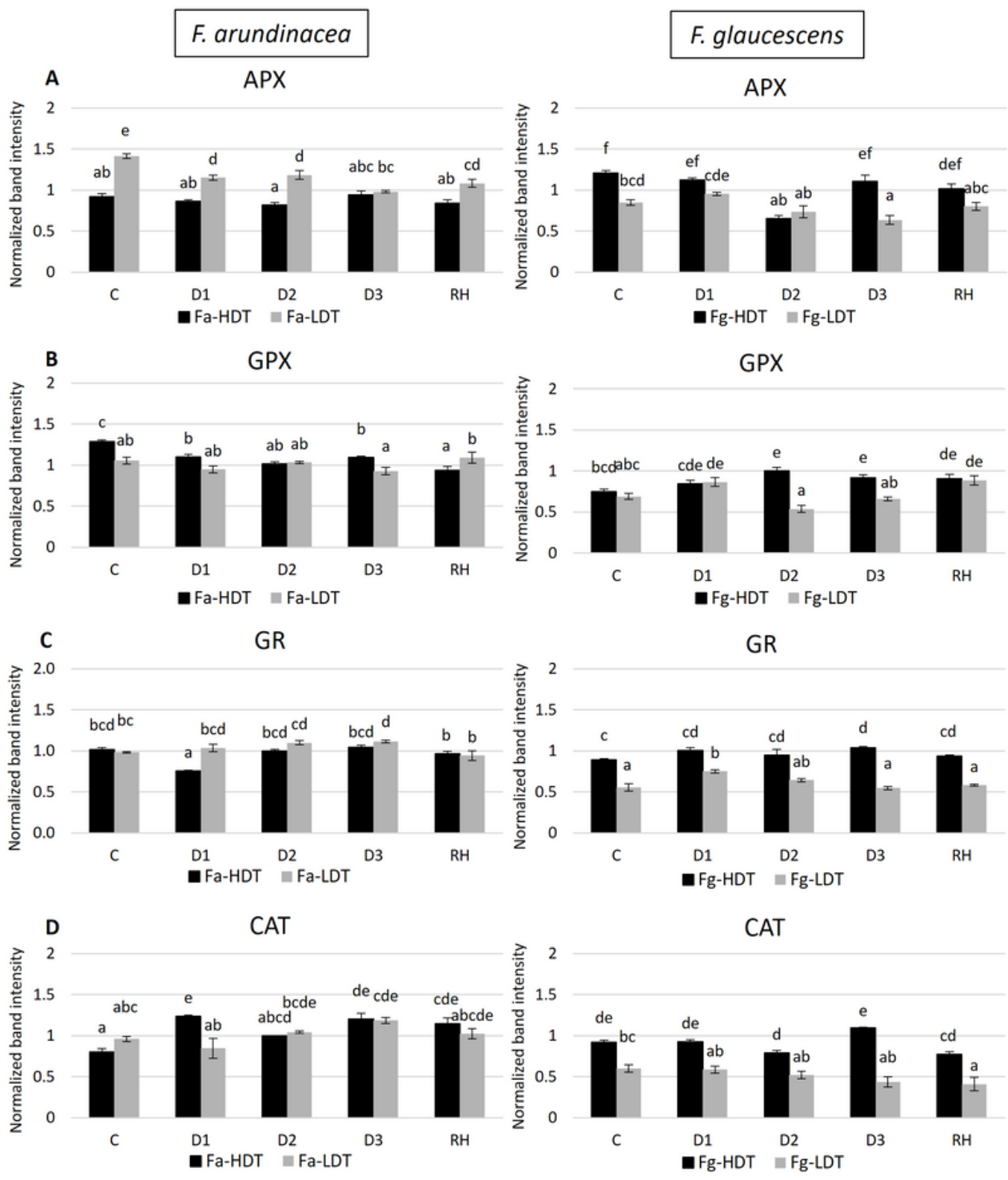
Figure 9

The protein level of L-ascorbate peroxidase (APX) (A), chloroplastic glutathione peroxidase (GPX) (B), glutathione reductase (GR) (C) and catalase (CAT) (D) in two genotypes of F. arundinacea (Fa-HDR, FaLDR) and F. glaucescens (Fg-HDR, Fg-LDR) before stress treatment (C), on the 3rd (D1), 6th (D2) and 11th (D3) day of water deficit and 10 days after subsequent re-watering (RH). Error bars represent the standard errors (SE) of three biological and two technical replicates. Homogeneity groups according to Fischer LSD test $(P=0.01)$ are denoted by the same letters.
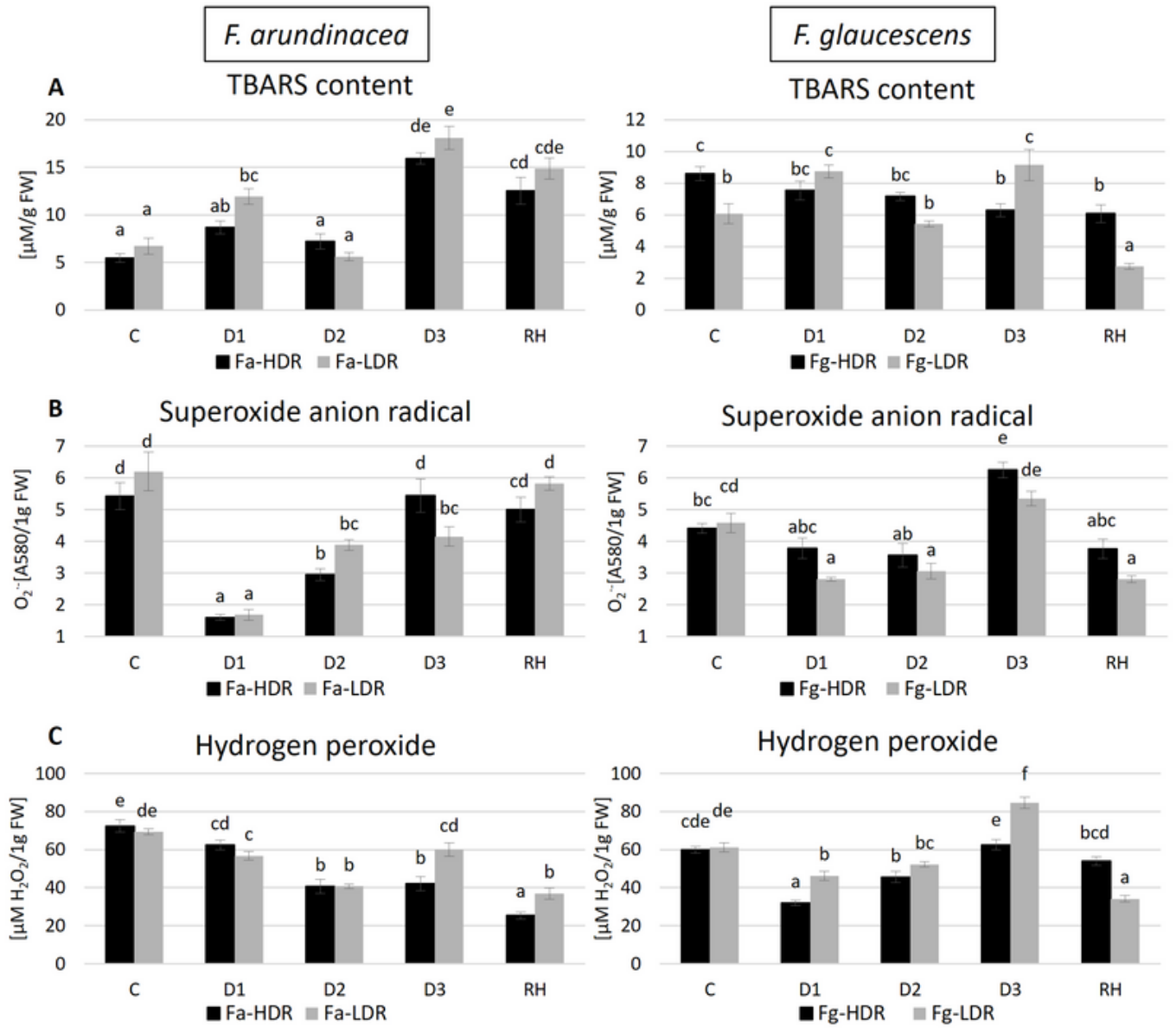
Figure 10

TBARS content (A), superoxide anion radical content (02--) (B), hydrogen peroxide content $(\mathrm{H} 2 \mathrm{O2})(\mathrm{C})$ in two genotypes of F. arundinacea (Fa-HDR, Fa-LDR) and F. glaucescens (Fg-HDR, Fg-LDR) before stress treatment (C), on the 3rd (D1), 6th (D2) and 11th (D3) day of water deficit and 10 days after subsequent re-watering (RH). Error bars represent the standard errors (SE) of three biological and two technical replicates. Homogeneity groups according to Fischer LSD test $(P=0.01)$ are denoted by the same letters.

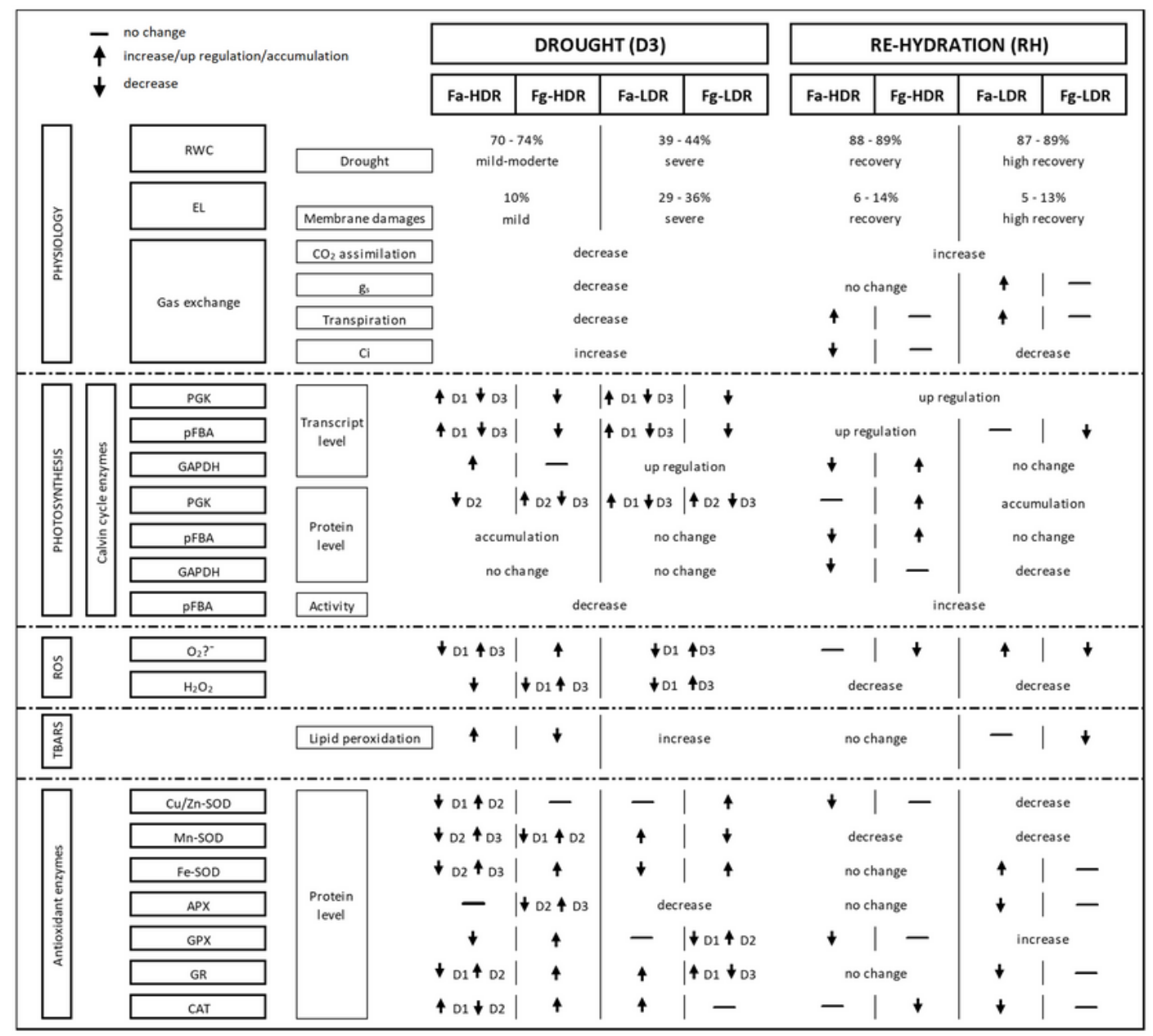


Figure 11

Comparison of physiological and molecular reactions in the HDR (Fa-HDR, Fg-HDR) and LDR (Fa-LDR, FgLDR) genotypes of F. arundinacea and F. glaucescens to drought stress on the 11th of water deficit (D3), unless otherwise stated; and after re-hydration (RH) in relation to D3.

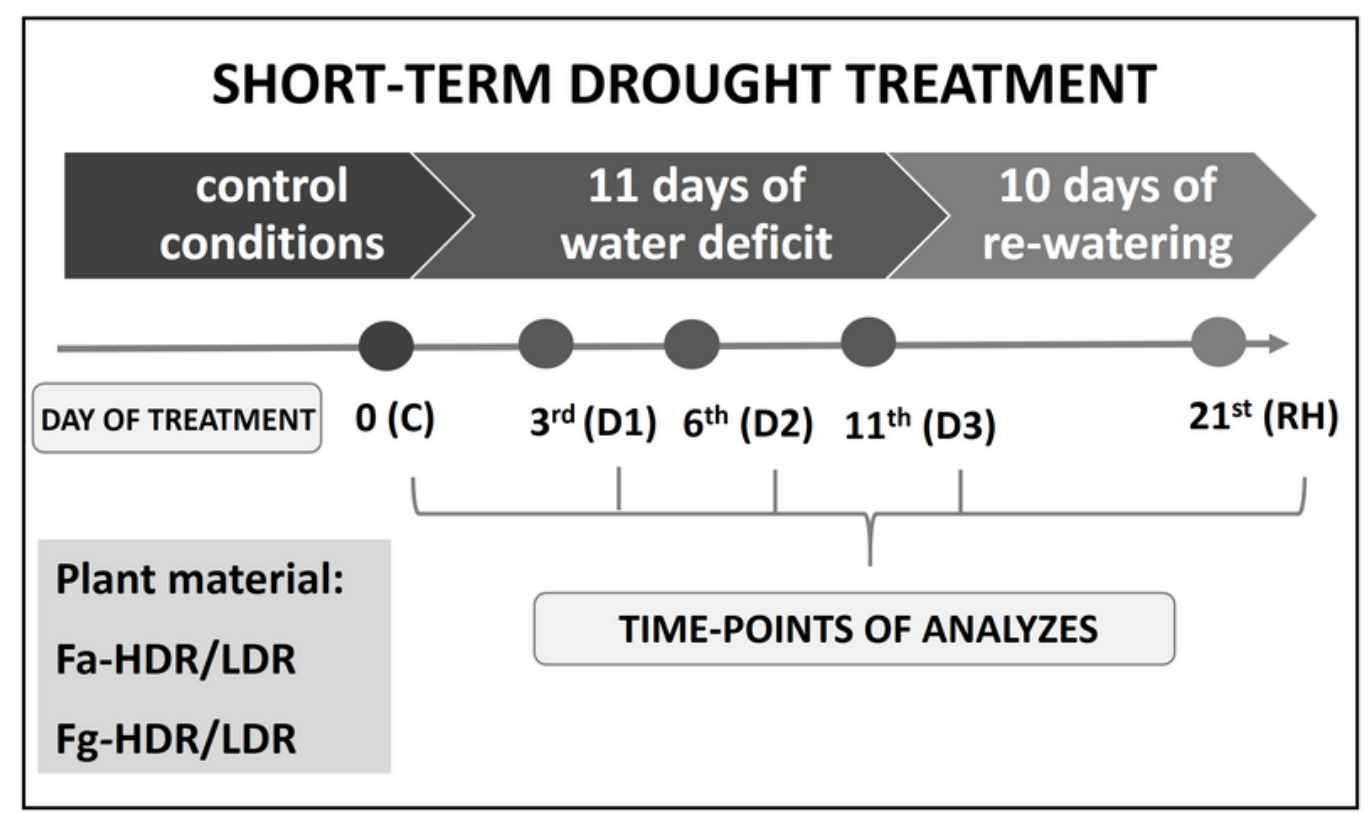

Figure 12 
The scheme of short-term drought experiment performed with F. arundinacea and F. glaucescens

\section{Supplementary Files}

This is a list of supplementary files associated with this preprint. Click to download.

- Fig.S1.png

- Fig.S2.png 